\title{
ON THE GEOMETRY OF RIEMANNIAN MANIFOLDS WITH A LIE STRUCTURE AT INFINITY
}

\author{
BERND AMMANN, ROBERT LAUTER, and VICTOR NISTOR
}

Received 16 December 2002

\begin{abstract}
We study a generalization of the geodesic spray and give conditions for noncomapct manifolds with a Lie structure at infinity to have positive injectivity radius. We also prove that the geometric operators are generated by the given Lie algebra of vector fields. This is the first one in a series of papers devoted to the study of the analysis of geometric differential operators on manifolds with Lie structure at infinity.
\end{abstract}

2000 Mathematics Subject Classification: 53C21, 53C27, 58J40.

1. Introduction. Geometric differential operators on complete noncompact Riemannian manifolds were extensively studied due to their applications to physics, geometry, number theory, and numerical analysis. Still, their properties are not as well understood as those of differential operators on compact manifolds, one of the main reasons being that differential operators on noncompact manifolds do not enjoy some of the most useful properties enjoyed by their counterparts on compact manifolds.

For example, elliptic operators on noncompact manifolds are not Fredholm in general. (We use the term "elliptic" in the sense that the principal symbol is invertible outside the zero section.) Also, one does not have a completely satisfactory pseudodifferential calculus on an arbitrary complete noncompact Riemannian manifold, which might allow us to decide whether a given geometric differential operator is bounded, Fredholm, or compact (see however [2] and the references therein).

However, if one restricts oneself to certain classes of complete noncompact Riemannian manifolds, one has a chance to obtain more precise results on the analysis of the geometric differential operators on those spaces. This paper is the first in a series of papers devoted to the study of such a class of Riemannian manifolds, the class of Riemannian manifolds with a "Lie structure at infinity" (see Definition 3.1). We stress here that few results on the geometry of these manifolds have a parallel in the literature, although there are a fair number of papers devoted to the analysis on particular classes of such manifolds $[12,13,15,16,17,38,39,55,57,64,67,70,74,75,76,78]$. The philosophy of Cordes' comparison algebras [14], Kondratiev's approach to analysis on singular spaces [38], Parenti's work on manifolds that are Euclidean at infinity [64], and Melrose's approach to pseudodifferential analysis on singular spaces [55] have played an important role in the development of this subject.

A manifold $M_{0}$ with a Lie structure at infinity has, by definition, a natural compactification to a manifold with corners $M=M_{0} \cup \partial M$ such that the tangent bundle $T M_{0} \rightarrow M_{0}$ 
extends to a vector bundle $A \rightarrow M$ with some additional structure. We assume, for example, that the Lie bracket of vector fields on $M_{0}$ defines, by restriction, a Lie algebra structure on the space of sections of $A$ such that the space $\mathscr{V}:=\Gamma(A)$ of sections of $A$ identifies with a Lie subalgebra of the Lie algebra of all vector fields on $M_{0}$. The pair $(M, \mathscr{V})$ then defines a Lie structure at infinity on $M_{0}$. A simple, nontrivial class of manifolds with a Lie structure at infinity is that of manifolds with cylindrical ends. Let $M_{0}$ be a manifold with cylindrical ends. In this case, the compactification $M$ is a manifold with boundary and $\mathscr{V}$ consists of all vector fields tangent to the boundary of $M$. This example plays a prominent role in the analysis of boundary value problems on manifolds with conical points [38, 40, 56, 58, 71, 72]. See the above references for earlier results.

Let $(M, \mathscr{V})$ be a Lie structure at infinity on $M_{0}, \mathscr{V}=\Gamma(A)$. The choice of a fiberwise scalar product on $A$ gives rise to a fiberwise scalar product $g$ on $T M_{0}$, that is, a Riemannian metric on $M_{0}$. Since $M$ is compact, any two such metrics $g_{1}$ and $g_{2}$ are equivalent in the sense that there exists a positive constant $C>0$ such that $C^{-1} g_{1} \geq g_{2} \geq C g_{1}$. One can thus expect that the properties of the Riemannian manifold $\left(M_{0}, g\right)$ obtained by the above procedure depend only on the Lie structure at infinity on $M_{0}$ and not on the particular choice of a metric on A. However, as shown in the following example, a metric on $M_{0}$ does not determine a Lie structure at infinity on $M_{0}$.

EXAMPLE 1.1. We compactify $\mathbb{R}$ by including $+\infty$ and $-\infty$ :

$$
\overline{\mathbb{R}}:=\mathbb{R} \cup\{+\infty,-\infty\} .
$$

We define $\varphi:[-1,+1] \rightarrow \overline{\mathbb{R}}, \varphi(t)=\log (t+1)-\log (1-t), \varphi( \pm 1)= \pm \infty$. The pullback of the differentiable structure on $[-1,1]$ defines a differentiable structure on $\overline{\mathbb{R}}$. On $\overline{\mathbb{R}}$ we consider the Lie algebra of vector fields that vanish at $\pm \infty$. The product of these compactifications of $\mathbb{R}$ defines a Lie structure on $M_{0}:=\mathbb{R}^{n}$, in which the compactification $M$ is diffeomorphic to the manifold with corners $[-1,1]^{n}$ and the sections of $A$ are all vector fields tangent to all hyperfaces. (The resulting Lie structure at infinity is that of the $b$-calculus (see Example 2.5)). Alternatively, one can consider the radial compactification of $\mathbb{R}^{n}$. The resulting Lie structure at infinity is described in Example 2.6, which is closely related to the so-called scattering calculus [57, 64].

We thus see that $\mathbb{R}^{n}$ fits into our framework and is in fact a manifold with a Lie structure at infinity for several distinct compactifications $M$.

Thus, although our motivation for studying manifolds with a Lie structure at infinity comes from analysis, this class of manifolds leads to some interesting questions about their geometry, and this paper (the first one in a series of papers on this subject $[2,3,4])$ is devoted mainly to the issues and constructions that have a strong Riemannian geometric flavor. It is important to mention here that only very few results on the geometry of particular classes of Riemannian manifolds with a Lie structure at infinity were proved before, except some special examples (e.g., compact manifolds and manifolds with cylindrical ends). For example, we prove that $M_{0}$ is complete and has bounded curvature in the sense that the Riemannian curvature $R$ and all its covariant derivatives $\nabla^{k} R$, with respect to the Levi-Civita connection, are bounded. Also, under 
some mild assumptions on $(M, \mathscr{V})$, we prove that $\left(M_{0}, g\right)$ has positive injectivity radius, and hence $M_{0}$ has bounded geometry. This is very convenient for the analysis on these manifolds. The main technique is based on generalizing the Levi-Civita connection to an " $A^{*}$-valued connection" on $A$. An $A^{*}$-valued connection on a bundle $E \rightarrow M$ is a differential operator $\nabla: E \rightarrow E \otimes A^{*}$ that satisfies all the usual properties of a connection, but with $A$ replacing the tangent bundle, see Definition 2.20. This concept was first introduced in a slightly different form in [25] by Evens et al. The right approach to the geometry of manifolds with a Lie structure at infinity requires the replacement of the tangent bundle by $A$. This was noticed before in particular examples, see, for instance, [12, 45, 55, 57, 59].

The Lie structure at infinity on $M_{0}$ allows us to define a canonical algebra of differential operators on $M_{0}$, denoted by Diff $(\mathscr{V})$, as the algebra of differential operators generated by the vector fields in $\mathscr{V}=\Gamma(A)$ and multiplication by functions in $\mathscr{C}^{\infty}(M)$. If $E_{0}, E_{1} \rightarrow M$ are vector bundles on $M$, then one can similarly define the spaces Diff $\left(\mathscr{V} ; E_{0}, E_{1}\right)$ (algebras if $E_{0}=E_{1}$ ) of differential operators generated by $\mathscr{V}$ and acting on sections of $E_{0}$ with values sections of $E_{1}$. All geometric operators on $M_{0}$ (de Rham, Laplace, and Dirac) will belong to one of the spaces Diff ( $\left.\mathscr{V} ; E_{0}, E_{1}\right)$, for suitable bundles $E_{0}$ and $E_{1}$. The proof of this result depends on our extension of the Levi-Civita connection to an $A^{*}$-valued connection.

Many questions on the analysis on noncompact manifolds or on the asymptotics of various families of operators can be expressed in terms of Diff $(\mathcal{V})$. We refer to $[16,18,32,42,43,45,50,53,55,57]$ for just a few of the many possible examples in the literature. Indeed, let $\Delta=d^{*} d \in$ Diff ( $\left.\mathscr{V}\right)$ be the scalar Laplace operator on $M_{0}$. Then $\Delta$ is essentially selfadjoint on $\mathscr{C}_{c}^{\infty}\left(M_{0}\right)$ by old results of Gaffney [26] and Roelcke [68]. Assume that $M_{0}$ has positive injectivity radius, then $P(1+\Delta)^{-m / 2}$ and $(1+\Delta)^{-m / 2} P$ are bounded operators on $L^{2}\left(M_{0}\right)$, for any differential operator $P \in \operatorname{Diff}(\mathcal{V})$ of order at most $m$. Cordes $[13,14]$ defined the comparison algebra $\mathcal{H}(M, \mathscr{V})$ as the norm-closed algebra generated by the operators $P(1+\Delta)^{-m / 2}$ and $(1+\Delta)^{-m / 2} P$, with $P \in \operatorname{Diff}(\mathscr{V})$ a differential operator of order at most $m$. The comparison algebra is useful because it leads to criteria for differential and pseudodifferential operators to be compact or Fredholm between suitable Sobolev spaces [2, 4, 42].

We expect manifolds with a Lie structure at infinity and especially the analytic tools (pseudodifferential and asymptotic analysis) that we have established in $[2,4]$ to play an important role for solving some problems in geometric analysis simultaneously for a large class of manifolds. Indeed, in special cases of manifolds with a Lie structure at infinity, the solutions to quite a few interesting problems in geometric analysis rely heavily on those methods. For instance, consider asymptotically Euclidean manifolds, a special case of Example 2.6. In general relativity, one is interested in finding solutions to the Einstein equations whose spatial part is asymptotically Euclidean. Integration of the first nontrivial coefficient in the asymptotic development of the metric at infinity yields the so-called "mass" of the solution [8]. The positive mass theorem states that any nonflat asymptotically Euclidean Riemannian manifold with nonnegative scalar curvature has positive mass. An elegant proof of the positive mass theorem by Witten (see [65]) uses Sobolev embeddings on such manifolds. The positive mass theorem provides the 
final step in the proof of the Yamabe conjecture on compact manifolds [69]: any conformal class on a compact manifold $M$ admits a metric with constant scalar curvature. In order to prove the conjecture in the locally conformally flat case, one replaces the metric $g$ on $M$ by a scalar-flat metric $u \cdot g$ on $M \backslash\{p\}$, where $u$ is a function $u(x) \rightarrow \infty$ for $x \rightarrow p$, and a neighborhood of $p$ provides the asymptotically Euclidean end, and one applies the positive mass conjecture to this. On most noncompact manifolds, the Yamabe problem is still unsolved. However, special cases have been solved, for example, on manifolds with cylindrical ends [1].

Both the geometry and the analysis of asymptotically hyperbolic manifolds have been the subject of articles on general relativity and the analysis of 3-manifolds, see [5, 6]. One can prove rigidity theorems [7] for asymptotically hyperbolic ends, or existence results for asymptotically hyperbolic Einstein metrics [47]. Similar rigidity problems for asymptotically complex hyperbolic ends are the subject of $[10,11,30]$.

Another example is the construction of manifolds with special holonomy $\mathrm{SU}(m)$, $\operatorname{Sp}(m)$, and $G_{2}$ where the analysis of weighted function spaces on manifolds which are quasi-asymptotically locally Euclidean [34, 35, 36] has been used.

In summary, our present program will lead to a unified approach to the analysis on various types of manifolds with a "good" asymptotic behavior at infinity.

We now discuss the contents of each section. In Section 2 we introduce and study structural Lie algebras of vector fields and the equivalent concept of boundary tangential Lie algebroids. A structural Lie algebra of vector fields on a manifold with corners $M$ gives rise to a canonical algebra Diff $(\mathscr{V})$ of differential operators. We include numerous examples.

Then, in Section 3, we specialize in the case that the constraints are only on the boundary. This special case is called a "manifold with a Lie structure at infinity." The Lie structure at infinity defines a Riemannian metric on the interior of the manifold. This metric is unique up to bi-Lipschitz equivalence. Hence, the Lie structure at infinity is a tool for studying a large class of open Riemannian manifolds. We are interested in the analysis on such open manifolds.

Section 4 is devoted to the study of the geometry of Riemannian manifolds with a Lie structure at infinity. We will prove that these manifolds are complete and have bounded curvature (together with all its covariant derivatives). This depends on an extension of the Levi-Civita connection to an $A^{*}$-valued connection, the appropriate notion of connection in this setting. Then we investigate the question of whether a Riemannian manifold with a Lie structure at infinity has positive injectivity radius.

In Section 5, we introduce Dirac and generalized Dirac operators and prove that they belong to Diff ( $\mathscr{V} ; W)$, where $W$ is a Clifford module. The same property is shared by all geometric operators (Laplace, de Rham, and signature) on the open manifold $M_{0}$.

2. Structural Lie algebras and Lie algebroids. We introduce in this section the concept of structural Lie algebras of vector fields, which is then used to define manifolds with a Lie structure at infinity.

2.1. Projective modules. In this subsection, we recall some well-known facts about projective modules over $\mathscr{C}^{\infty}(M)$, where $M$ is a compact manifold, possibly with corners. 
Let $V$ be a $\mathscr{C}^{\infty}(M)$-module with module structure $\mathscr{C}^{\infty}(M) \times V \ni(f, v) \mapsto f v \in V$. Let $x \in M$ and denote by $\mathfrak{p}_{x}$ the set of functions on $M$ that vanish at $x \in M$. Then $\mathfrak{p}_{x} V$ is a complex vector subspace of $V$ and $V / p_{x} V$ is called the geometric fiber of $V$ at $x$. In general, the geometric fibers of $V$ are complex vector spaces of varying dimensions.

A subset $S \subset V$ will be called a basis of $V$ if every element $v \in V$ can be written

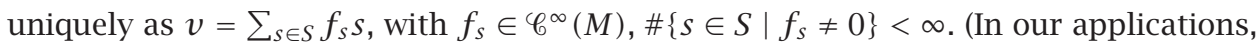
$S$ will always be a finite set, so we will not have to worry about this last condition.) A $\mathscr{C}^{\infty}(M)$-module is called free (with basis $S$ ) if it has a basis $S$. Unlike the general case, the geometric fibers of a free module have constant dimension, equal to the number of elements in the basis $S$. Note, however, that if $f: V \rightarrow W$ is a morphism of free modules, the induced map between geometric fibers may have nonconstant rank. For example, it is possible that $f$ is injective, but the induced map on the geometric fibers is not injective on all fibers. An example is provided by $M=[0,1], V=W=\mathscr{C}^{\infty}([0,1])$, and $f$ given by the multiplication by the coordinate function $x \in[0,1]$. Then $f$ is injective, but the induced map on the geometric fibers at 0 is 0 .

A $\mathscr{C}^{\infty}(M)$-module $V$ is called finitely generated projective if, by definition, there exists another module $W$ such that $V \oplus W$ is free with a finite basis. We then have the following fundamental theorem of Serre and Swan (see [37]).

THEOREM 2.1 (Serre and Swan). If $V$ is a finitely generated projective module over $\mathscr{C}^{\infty}(M)$, then the set $E:=\cup_{x \in M}\left(V / \mathfrak{p}_{x} V\right) \times\{x\}$, the disjoint union of all geometric fibers of $V$, can be endowed with the structure of a finite-dimensional, smooth vector bundle $E \rightarrow M$ such that $V \simeq \Gamma(M ; E)$. The converse is also true: $\Gamma(M ; E)$ is a finitely generated projective $\mathscr{C}^{\infty}(M)$-module for any finite-dimensional, smooth vector bundle $E \rightarrow M$.

Suppose now that $V$ is a $\mathscr{C}^{\infty}(M)$-module and that $M$ is connected. Then $V$ is a finitely generated projective $\mathscr{C}^{\infty}(M)$-module if and only if there exists $k \in \mathbb{Z}_{+}$satisfying the following condition: for any $x \in M$, there exist $\varphi \in \mathscr{C}^{\infty}(M), \varphi(x)=1$, and $k$-elements $v_{1}, \ldots, v_{k} \in V$ with the property that, for any $w \in V$, we can find $f_{1}, f_{2}, \ldots, f_{k} \in \mathscr{C}^{\infty}(M)$ such that

$$
\varphi\left(f_{1} v_{1}+f_{2} v_{2}+\cdots+f_{k} v_{k}-w\right)=0 \text { in } V
$$

and, moreover, the germs of $f_{1}, \ldots, f_{k}$ at $x$ are uniquely determined. A module $V$ satisfying condition (2.1) above is called locally free of rank $k$, and what we are saying here is that "locally free of rank $k$, for some $k$," is equivalent to "finitely generated projective." It is crucial here that the number of elements $k$ is the same for any $x \in M$. In case $M$ is not connected, the number $k$ needs only to be constant on the connected components of $M$.

REMARK 2.2. The introduction of projective modules over $C^{\infty}(M)$ in partial differential operators on noncompact manifolds was pioneered by Melrose [54] in the early 1980s.

2.2. Manifolds with corners and structural Lie algebras. We now fix our terminology and recall the definitions of the main concepts related to manifolds with corners. 
In the following, a manifold will always stand for a $C^{\infty}$-manifold possibly with corners. In contrast, a smooth manifold is a $C^{\infty}$-manifold without corners. By definition, for every point $p$ in a manifold with corners $M$, there are a coordinate neighborhood $U_{p}$ of $p$ and diffeomorphism $\varphi_{p}$ to $[0, \infty)^{k} \times \mathbb{R}^{n-k}$, with $\varphi_{p}(p)=0$ such that the transition functions are smooth (including on the boundary). The number $k$ here clearly depends on $p$, and will be called the boundary depth of $p$. Hence points in the interior have boundary depth 0 , points on the boundary of a manifold without corners have boundary depth 1 , and so forth. Roughly speaking, the boundary depth counts the number of boundary faces $p$ is in.

Moreover, we assume that each hyperface $H$ of $M$ is an embedded submanifold and has a defining function, that is, there exists a smooth function $x_{H} \geq 0$ on $M$ such that

$$
H=\left\{x_{H}=0\right\}, \quad d x_{H} \neq 0 \quad \text { on } H .
$$

This assumption is just a simplifying assumption. We can deal with general manifolds with corners using the constructions from [59]. Note that a priori we do not fix a particular system of defining functions, but only use their existence occasionally.

If $F \subset M$ is an arbitrary face of $M$ of codimension $k$, then $F$ is an open component of the intersection of the hyperfaces containing it. Any set $x_{1}, \ldots, x_{k}$ of defining functions of the hyperfaces containing $F$ is called a set of defining functions of $F$; thus, $F$ is a connected component of $\left\{x_{1}=x_{2}=\cdots=x_{k}=0\right\}$. This statement obviously does not depend on the choice of the defining functions $x_{j}, j=1, \ldots, k$. We will denote by $\partial M$ the union of all nontrivial faces of $M$. Usually, we will denote by $M_{0}$ the interior of $M$, that is, $M_{0}:=M \backslash \partial M$.

A submersion $f: M \rightarrow N$, between two manifolds with corners $M$ and $N$, is a differentiable map $f$ such that $d f$ is surjective at all points and $d f(v)$ is an inward pointing tangent vector of $N$ if and only if $v$ is an inward pointing vector $M$. It follows then that the fibers $f^{-1}(y)$ of $f$ are smooth manifolds without corners and that $f$ preserves the boundary depth (i.e., the number of boundary faces a point $p$ is in.) If $x$ is a defining function of some hyperface of $N$, then $x_{1}=x \circ f$ is such that $\left\{x_{1}=0\right\}$ is a union of hyperfaces of $M$ and $d x_{1} \neq 0$ on $\left\{x_{1}=0\right\}$.

EXAMPLE 2.3. Let $A \rightarrow M$ be a smooth vector bundle. The sphere bundle of $A$, denoted by $S(A)$, is defined, as usual, as the set of (positive) rays in the bundle $A$, that is, $S(A)=$ $(A \backslash\{0\}) / \mathbb{R}_{+}$. If we fix a smooth metric on $A$, then $S(A)$ identifies with the set of vectors of length one in $A$. Moreover, $S(A) \rightarrow M$ turns out to be a submersion of manifolds with corners.

A submanifold with corners $N$ of a manifold with corners $M$ is a submanifold $N \subset M$ such that $N$ is a manifold with corners, and each hyperface $H$ of $N$ is a connected component of a set of the form $H^{\prime} \cap N$, where $H^{\prime}$ is a hyperface of $M$ intersecting $N$ transversally.

The starting point of our analysis is a Lie algebra of vector fields on a manifold with corners. For reasons that will be clearer later, we prefer to keep this concept as general as possible, even if for the analysis on noncompact manifolds, only certain classes of Lie algebras of vector fields will be used. 
DEFINITION 2.4. A structural Lie algebra of vector fields on a manifold $M$ ( $M$ possibly with corners) is a subspace $\mathscr{V} \subset \Gamma(T M)$ of the real vector space of vector fields on $M$ with the following properties:

(i) $\mathscr{V}$ is closed under Lie brackets;

(ii) $\mathscr{V}$ is a finitely generated projective $\mathscr{C}^{\infty}(M)$-module;

(iii) the vector fields in $\mathscr{V}$ are tangent to all faces in $M$.

By (ii) we mean that $\mathscr{V}$ is closed for multiplication by functions in $\mathscr{C}^{\infty}(M)$ and the induced $\mathscr{C}^{\infty}(M)$-module structure makes it a finitely generated projective $\mathscr{C}^{\infty}(M)$-module.

Given a structural Lie algebra $\mathscr{V}$ of vector fields on a manifold with corners, we call the enveloping algebra Diff $(\mathscr{V})$ of $\mathscr{V}$ the algebra of $\mathscr{V}$-differential operators on $M$. Note that any $\mathscr{V}$-differential operator $P \in \operatorname{Diff}(\mathscr{V})$ can be realized as a polynomial in vector fields in $\mathscr{V}$ with coefficients in $\mathscr{C}^{\infty}(M)$ acting on the space $\mathscr{C}^{\infty}(M)$.

We give some examples for structural Lie algebras of vector fields. Some of these examples can also be found in [57]. We also give descriptions of the structural vector fields in local coordinates, because this will be helpful in the applications of the theory developed here. All of the following examples model the analysis on some noncompact manifold, except for the last one, which models the analysis of adiabatic families.

The following example is the simplest and most studied so far; however, it is quite important for us because it models the geometry of manifolds with cylindrical ends, and hence it is easier to grasp.

EXAMPLE 2.5. Let $M$ be a manifold with corners and

$$
\mathscr{V}_{b}=\{X \in \Gamma(T M): X \text { is tangent to all faces of } M\} .
$$

Then $\mathscr{V}_{b}$ is a structural Lie algebra of vector fields, and any structural Lie algebra of vector fields on $M$ is contained in $\mathscr{V}_{b}$, by condition (iii) of the above definition. A vector field $X \in \mathscr{V}_{b}$ is called a $b$-vector field $X$. Fix $x_{1}, \ldots, x_{k}$ and $y \in \mathbb{R}^{n-k}$ local coordinates near a point $p$ on a boundary face of codimension $k$, with $x_{j}$ defining functions of the hyperfaces through $p$. Then any $b$-vector field $X$ is of the form

$$
X=\sum_{j=1}^{k} a_{j}(x, y) x_{j} \partial_{x_{j}}+\sum_{j=1}^{n-k} b_{j}(x, y) \partial_{y_{j}}
$$

on some neighborhood of $p$, with the coefficients $a_{j}$ and $b_{j}$ smooth everywhere (including the hyperfaces $x_{j}=0$ ), for all $j$. This shows that the Lie algebra of $b$-vector fields is generated in a neighborhood $U$ of $p$ by $x \partial_{x}$ and $\partial_{y}$ as a $\mathscr{C}^{\infty}(M)$-module. The differential operators in Diff $\left(\mathscr{V}_{b}\right)$ are called Fuchs-type operators, totally characteristic, or simply, and perhaps more systematically, $b$-differential operators. The structural Lie algebra $\mathscr{V}_{b}$ and the analysis of the corresponding differential and pseudodifferential operators are treated in detail for instance in [20, 23, 33, 48, 56, 57, 73].

EXAMPLE 2.6. Let $M$ be a compact manifold with boundary and $x: M \rightarrow \overline{\mathbb{R}}_{+}$a boundary defining function. Then the Lie algebra $\mathscr{V}_{s c}:=x \mathscr{V}_{b}$ does not depend on the choice of $x$ and the vector fields in $\mathscr{V}_{s c}$ are called scattering vector fields; with respect to local coordinates $(x, y)$ near the boundary, scattering vector fields are generated by 
$x^{2} \partial_{x}$ and $x \partial_{y}$. An analysis of the scattering structure can be found in [57]. Since this structure models the analysis on asymptotically Euclidean spaces, let us be a little bit more precise and recall some basic definitions. A Riemannian metric $g$ on the interior of $M$ is called a scattering metric if, close to the boundary $\partial M$, it is of the form $g=d x^{2} / x^{4}+h / x^{2}$, where $h$ is a smooth, symmetric 2-tensor on $M$ which is nondegenerate when restricted to the boundary. Then scattering vector fields are exactly those smooth vector fields on $M$ that are of bounded length with respect to $g$, and the corresponding Laplacian $\Delta_{g}$ is an elliptic polynomial in scattering vector fields. As a special case of this setting, note that the radial compactification map

$$
\mathrm{RC}: \mathbb{R}^{N} \longrightarrow S_{+}^{N}:=\left\{\omega=\left(\omega_{0}, \omega^{\prime}\right) \in S^{N}: \omega_{0} \geq 0\right\}: z \longmapsto\left(1+|z|^{2}\right)^{-1 / 2}(1, z)
$$

identifies the Euclidean space $\mathbb{R}^{N}$ with the interior of the upper half-sphere $S_{+}^{N}$ such that the Euclidean metric lifts to a scattering metric on $S_{+}^{N}$.

The following example is one of the examples that we are interested in using in applications.

EXAMPLE 2.7. Let $M$ be a manifold with boundary $\partial M$, which is the total space of a fibration $\pi: \partial M \rightarrow B$ of smooth manifolds. We let

$$
\mathscr{V}_{e}=\{X \in \Gamma(T M): X \text { is tangent to all fibers of } \pi \text { at the boundary }\}
$$

be the space of edge vector fields. In order to show that this is indeed a structural Lie algebra of vector fields, we have to show that it is closed under Lie brackets. Let $i: \partial M \rightarrow M$ be the inclusion. Assume that $X, Y \in \mathscr{V}_{e}$. Because

$$
\left.[X, Y]\right|_{\partial M}=\left[\left.X\right|_{\partial M},\left.Y\right|_{\partial M}\right]
$$

the commutator is again tangent to the fibers of $\pi$. If $(x, y, z)$ are coordinates in a local product decomposition near the boundary, where $x$ corresponds to the boundary defining function, $y$ corresponds to a set of variables on the base $B$ lifted through $\pi$, and $z$ is a set of variables in the fibers of $\pi$, then edge vector fields are generated by $x \partial_{x}$, $x \partial_{y}$, and $\partial_{z}$. Using this local coordinate description is another way to see immediately that the space of edge vector fields is in fact a Lie algebra. More importantly, it shows that it is a projective $\mathscr{C}^{\infty}(M)$-module. The analysis of the Lie algebra $\mathscr{V}_{e}$ is partly carried out in [50] and more recently in [45].

A special case of the edge structure is of particular importance for the analysis on hyperbolic space, so it deserves its own name.

EXAMPLE 2.8. Let $M$ be a compact manifold with boundary and let $\mathscr{V}_{0}$ be the edge vector fields corresponding to the trivial fibration $\pi=\mathrm{id}: \partial M \rightarrow \partial M$, that is, we have

$$
\mathscr{V}_{0}=\left\{X \in \Gamma(T M):\left.X\right|_{\partial M}=0\right\}
$$


which explains the name 0-vector fields for the elements in $\mathscr{V}_{0}$. With respect to local coordinates $(x, y)$ near the boundary, 0 -vector fields are generated by $x \partial_{x}$ and $x \partial_{y}$. Recall that a Riemannian manifold $\left(M_{0}, g_{0}\right)$ is called conformally compact provided it is isometric to the interior of a compact manifold $M$ with boundary equipped with a metric $g=\varrho^{-2} h$ in the interior, where $h$ is a smooth metric on $M$ and $\varrho: M \rightarrow \overline{\mathbb{R}}_{+}$a boundary defining function. Note that 0 -vector fields are the smooth vector fields on $M$ that are of bounded length with respect to $g$; moreover, the Laplacian $\Delta_{g_{0}}$ is given as an elliptic polynomial in 0-vector fields. A particular example of conformally compact spaces is of course the hyperbolic space with compactification given by the ball model. Conformally compact spaces arise naturally in questions related to the Einstein equation [5, 47, 52], and the "AdS/CFT-correspondence." An analysis of 0-vector fields and the associated 0differential and pseudodifferential operators was carried out for instance in $[41,50,66]$. Criteria for the Fredholmness of operators in Diff $\left(\mathscr{V}_{0}\right)$, which is crucial in the approach to the study of Einstein's equations on conformally compact manifolds used in the above-mentioned papers, were established for instance in [41, 42, 44, 45, 50, 51, 57, 66].

The structural Lie algebra of vector fields in the next example is a slight variation of the Lie algebra of edge vector fields; however, it is worth pointing out that this slight variation leads to a completely different analysis for the associated (pseudo)differential operators.

EXAMPLE 2.9. Let $M$ be as in Example 2.7 and let $x: M \rightarrow \overline{\mathbb{R}}_{+}$be a boundary defining function. Then $\mathscr{V}_{d e}:=x \mathscr{V}_{e}$ is a structural Lie algebra of vector fields; the corresponding structure is called the double-edge structure. With respect to local product coordinates as in Example 2.7, double-edge vector fields are generated by $x^{2} \partial_{x}, x^{2} \partial_{y}$, and $x \partial_{z}$. The analysis of the double-edge structure, which is in fact much simpler than the corresponding analysis of the edge structure, can be found for instance in [43].

The following example appears in the analysis of holomorphic functions of several variables.

EXAMPLE 2.10. Let $M$ be a smooth compact manifold with boundary $\partial M$ and let $\Theta \in \mathscr{C}^{\infty}\left(M, \Lambda^{1} T^{*} M\right)$ be a smooth 1 -form such that $i^{*} \Theta \neq 0$, where $i: \partial M \hookrightarrow M$ is the inclusion of the boundary. Moreover, let $x$ be a boundary defining function. Then

$$
\mathscr{V}_{\Theta}:=\left\{V \in \mathscr{V}_{b}: V=0 \text { at } \partial M, \Theta(V) \in x^{2} \mathscr{C}^{\infty}(M)\right\}
$$

is a structural Lie algebra of vector fields called the $\Theta$-structure. For a local description as well as for an analysis of the $\Theta$-structure, we refer to [24].

All the above examples of structural Lie algebras of vector fields model the analysis on certain noncompact manifolds (giving rise to algebras of differential operators that replace the algebra of totally characteristic differential operators) on manifolds with cylindrical ends. The following example, however, models the analysis of a family of adiabatic differential operators.

EXAMPLE 2.11. Let $N$ be a closed manifold that is the total space of a locally trivial fibration $\pi: N \rightarrow B$ of closed manifolds, let $T N / B \rightarrow N$ be the vertical tangent bundle, 
and let $M:=N \times[0, \infty)_{x}$. Then

$$
\mathscr{V}_{a}:=\{V \in \Gamma(T M): V(x) \in T N \forall x \in[0, \infty), V(0) \in \Gamma(T N / B)\}
$$

is a structural Lie algebra of vector fields called the adiabatic algebra. If $(y, z)$ are local coordinates on $N$, where again the set of variables $y$ corresponds to variables on the base $B$ lifted through $\pi$, and $z$ are variables in the fibers, then adiabatic vector fields are generated by $x \partial_{y}$ and $\partial_{z}$. The adiabatic structure has been studied and used for instance in $[60,61]$.

We will sometimes refer to a structural Lie algebra of vector fields simply as Lie algebra of vector fields, when no confusion can arise. Because $\mathscr{V}$ is a finitely generated projective $\mathscr{C}^{\infty}(M)$-module, using the Serre-Swan theorem [37] (recalled above, see Theorem 2.1), we obtain that there exists a vector bundle

$$
A=A_{\mathscr{V}} \longrightarrow M \text { such that } \mathscr{V} \cong \Gamma\left(A_{\mathscr{V}}\right)
$$

naturally as $\mathscr{C}^{\infty}(M)$-modules. We will identify from now on $\mathscr{V}$ with $\Gamma\left(A_{\mathscr{V}}\right)$. The following proposition is due to Melrose [54].

Proposition 2.12. If $\mathscr{V}$ is a structural Lie algebra of vector fields, then there exists a natural vector bundle map $\varrho: A_{\mathscr{V}} \rightarrow T M$ such that the induced map $\varrho_{\Gamma}: \Gamma\left(A_{\mathscr{V}}\right) \rightarrow \Gamma(T M)$ identifies with the inclusion map.

Proof. Let $m \in M$. Then the fiber $A_{m}$ at $m$ of $A=A_{\mathscr{V}} \rightarrow M$ identifies with $\mathscr{V} / \mathfrak{p}_{m} \mathscr{V}$, where $\mathfrak{p}_{m}$ is the ideal of $\mathscr{C}^{\infty}(M)$ consisting of smooth functions on $M$ that vanish at $m$. Recall now that $\mathscr{V}$ consists of vector fields on $M$. Then the map $A_{m} \rightarrow T_{m} M$ sends the class of $X \in \mathscr{V}$ to the vector $X(m) \in T_{m} M$.

REMARK 2.13. The condition in Definition 2.4 that $\mathscr{V}$ has to be projective is essential. As an example, consider $M=[0,1]$ and let

$$
\begin{aligned}
\mathscr{V}:= & \left\{f(t) \partial_{t}: f:[0,1] \longrightarrow \mathbb{R} \text { smooth, } f(1)=0,\right. \\
& \left.t^{k}\left(d^{m} f / d t^{m}\right) \longrightarrow 0 \text { as } t \rightarrow 0 \forall k, m \in \mathbb{N} \cup\{0\}\right\} .
\end{aligned}
$$

Then $\mathscr{V}$ is a $\mathscr{C}^{\infty}(M)$-module. However, $\mathscr{V}$ is not a projective $\mathscr{C}^{\infty}(M)$-module, as we can see by contradiction. Assume $\mathscr{V}$ were projective. Then there is a bundle $A$ over $[0,1]$ with $\mathscr{V}=\Gamma(A)$. Let $s$ be a trivialization of $A$, that is, $s(t)=f(t) \partial_{t}$ with $f$ as above. Hence $\tilde{f}(t)=(1 / t) f(t)$ also decays sufficiently fast; however,

$$
\Gamma(A) \not \frac{1}{t} s(t)=\tilde{f}(t) \partial_{t} \in \mathscr{V} .
$$

It is convenient for the following discussion to recall the definition of a Lie algebroid. General facts about Lie algebroids can be found in [18, 49] (a few basic facts are also summarized in [63]). 
Definition 2.14. A Lie algebroid $A$ over a manifold $M$ is a vector bundle $A$ over $M$, together with a Lie algebra structure on the space $\Gamma(A)$ of smooth sections of $A$ and a bundle map $\varrho: A \rightarrow T M$, extended to a map $\varrho_{\Gamma}: \Gamma(A) \rightarrow \Gamma(T M)$ between sections of these bundles, such that

(i) $\varrho_{\Gamma}([X, Y])=\left[\varrho_{\Gamma}(X), \varrho_{\Gamma}(Y)\right]$,

(ii) $[X, f Y]=f[X, Y]+\left(\varrho_{\Gamma}(X) f\right) Y$, for any smooth sections $X$ and $Y$ of $A$ and any smooth function $f$ on $M$.

The map $\varrho_{\Gamma}$ is called the anchor of $A$. If, in addition,

(iii) all vector fields $\varrho_{\Gamma}(\Gamma(A))$ are tangential to the faces, then the Lie algebroid $A \rightarrow M$ is called a boundary tangential Lie algebroid.

We thus see that there exists an equivalence between the concept of a structural Lie algebra of vector fields $\mathscr{V}=\Gamma\left(A_{\mathscr{V}}\right)$ and the concept of a boundary tangential Lie algebroid $\varrho: A \rightarrow T M$ such that $\varrho_{\Gamma}: \Gamma(A) \rightarrow \Gamma(T M)$ is injective and has range in $\mathscr{V}_{b}$. In order to shorten our notation, we will write $X f$ instead of $\varrho_{\Gamma}(X) f$ for the action of the sections of a Lie algebroid on functions if the meaning is clear from the context.

2.3. Constructing new Lie algebroids from old ones. Let $f: N \rightarrow M$ be a submersion of manifolds with corners in the above sense (which implies in particular that any fiber is a smooth manifold). Let $A=A_{\mathscr{V}}$ be a boundary tangential Lie algebroid over $M$.

DeFINITION 2.15. The thick pullback $f^{\#} A$ is the vector bundle over $N$ which at the point $p \in N$ is defined to be

$$
f^{\#} A_{p}:=\left\{(v, w) \mid v \in A_{f(p)}, w \in T_{p} N, f_{*}(w)=\varrho(v)\right\}
$$

equipped with the vector bundle structure induced by $f^{*}(A) \oplus T N$.

Projection to the first component yields a surjective linear map $f^{\#} A_{p} \rightarrow A_{f(p)}$, denoted in the following by $f_{*}$, and projection onto the second component yields a linear map $f^{\#} A_{p} \rightarrow T_{p} N$, denoted by $f^{\#} \varrho$.

We obtain the commuting diagram

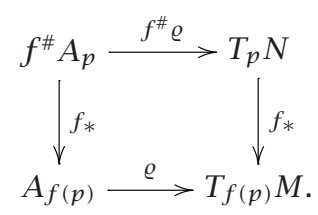

For example, $f^{\#} T M=T N$.

LEMMA 2.16. The thick pullback $f^{\#} A$ is a boundary tangential Lie algebroid over $N$ with anchor map given by $f^{\#} \varrho$.

Proof. Let $\Gamma_{\text {vert }} T N$ denote the bundle of vertical sections $X$, that is, $f_{*} X=0$. This bundle coincides by definition with the analogously defined bundle of vertical sections 
of $A$. The rows of the following commutative diagram are exact:

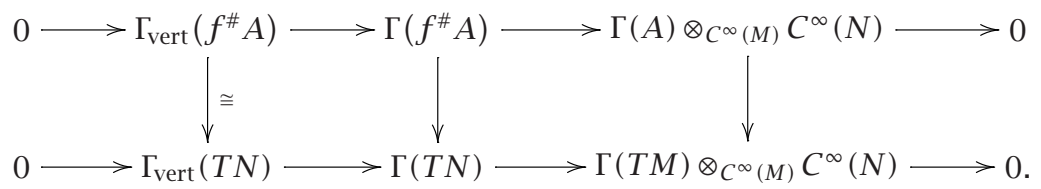

The vertical arrows are inclusions. The horizontal arrows of the second row are Lie algebra homomorphisms. The space $\Gamma(A)$ is by definition a Lie subalgebra of $\Gamma(T M)$, thus $\Gamma(A) \otimes_{C^{\infty}(M)} C^{\infty}(N)$ is a Lie subalgebra of $\Gamma(T M) \otimes_{C^{\infty}(M)} C^{\infty}(N)$. A standard diagram chase then implies that $\Gamma\left(f^{\#} A\right)$ is also a Lie subalgebra of $\Gamma(T N)$.

The fact that $A$ is projective (resp., boundary tangential) immediately implies that $\Gamma\left(f^{\#} A\right)$ is also projective (resp., boundary tangential).

Let $\mathfrak{g}$ and $\mathfrak{h}$ be two Lie algebras. Suppose that there is given an action by derivations of $\mathfrak{g}$ on $\mathfrak{h}$ :

$$
\varphi: \mathfrak{g} \longrightarrow \operatorname{Der}(\mathfrak{h})
$$

Then we can define the semidirect sum $\mathfrak{g} \ltimes_{\varphi} \mathfrak{h}$ as follows. As a vector space, $\mathfrak{g} \ltimes_{\varphi} \mathfrak{h}=\mathfrak{g} \oplus \mathfrak{h}$, and the Lie bracket is given by

$$
\left[\left(X_{1}, Y_{1}\right),\left(X_{2}, Y_{2}\right)\right]:=\left(\left[X_{1}, X_{2}\right], \varphi_{X_{1}}\left(Y_{2}\right)-\varphi_{X_{2}}\left(Y_{1}\right)+\left[Y_{1}, Y_{2}\right]\right)
$$

for any $X_{1}, X_{2} \in \mathfrak{g}$ and $Y_{1}, Y_{2} \in \mathfrak{h}$. We will usually omit the index $\varphi$ denoting the action by derivations in the notation for the semidirect sum.

We want to use this construction to obtain new Lie algebroids from old ones. Assume then that we are given two Lie algebroids $A, L \rightarrow M$ over the same manifold and that $\Gamma(A)$ acts by derivations on $\Gamma(L)$. Denote this action by $\varphi$, as above. We assume that the action of $\Gamma(A)$ on $\Gamma(L)$ is compatible with the $\mathscr{C}^{\infty}(M)$-module structure on $\Gamma(L)$ in the sense that

$$
\varphi_{X}(f Y)=X(f) Y+f \varphi_{X}(Y)
$$

for any $X \in \Gamma(A), Y \in \Gamma(L)$, and $f \in \mathscr{C}^{\infty}(M)$. Assume, for simplicity, that the structural map (anchor) $L \rightarrow T M$ is zero, then we can endow $\Gamma(A \oplus L)=\Gamma(A) \oplus \Gamma(L)$ with the semidirect sum structure obtained from $\Gamma(A) \ltimes \Gamma(L)$ such that $A \oplus L$ becomes a Lie algebroid denoted by $A \ltimes L$, and called the semidirect product of $L$ by $A$ [31]. Thus

$$
\Gamma(A \ltimes L)=\Gamma(A) \ltimes \Gamma(L) .
$$

In the language of Lie algebroids, the action of $\Gamma(A)$ on $\Gamma(L)$ considered above is called a representation of $A$ on $L$. In a similar way, the action of $\Gamma(A)$ on $\Gamma(L)$ by derivation, considered above, deserves to and will be called a representation by derivations of $A$ on $L$. If $A \rightarrow M$ is a tangential Lie algebroid, then $A \ltimes L \rightarrow M$ will also be one. 
2.4. Differential operators. From now on we will assume that $A$ denotes the vector bundle determined by the structural Lie algebra $\mathscr{V}$ and vice versa.

DEFINITION 2.17. Let Diff $(\mathscr{V})$ denote the algebra of differential operators generated by $\mathscr{V}$, where the vector fields are regarded as derivations on functions.

We also want to study differential operators with coefficients in vector bundles. Let $E_{1} \rightarrow M$ and $E_{2} \rightarrow M$ be two vector bundles. Embed $E_{i} \hookrightarrow M \times \mathbb{C}^{N_{i}}, i=1,2$. Denote by $e_{i}$ a projection in $M_{N_{i}}\left(\mathscr{C}^{\infty}(M)\right)$ whose (pointwise) range is $E_{i}$. Then we define

$$
\operatorname{Diff}\left(\mathscr{V} ; E_{1}, E_{2}\right):=e_{2} M_{N_{2} \times N_{1}}(\operatorname{Diff}(\mathscr{V})) e_{1}
$$

This definition of Diff ( $\left.\mathscr{V} ; E_{1}, E_{2}\right)$ is independent of the choices of the embeddings $E_{i} \hookrightarrow$ $M \times \mathbb{C}^{N_{i}}$ and of the choice of $e_{i}$. Elements of Diff $\left(\mathscr{V} ; E_{0}, E_{1}\right)$ will be called differential operators generated by $\mathscr{V}$. In the special case $E_{1}=E_{2}=E$, we simply write Diff $(\mathscr{V} ; E)$, the algebra of differential operators on $E$ generated by $\mathscr{V}$.

It is possible to describe the differential operators in Diff $(\mathscr{V} ; E)$ locally on $M$ as follows.

LEMмA 2.18. A linear map $D: \Gamma(E) \rightarrow \Gamma(E)$ is in Diff $(\mathscr{V} ; E)$ if and only if, for any trivialization $\left.E\right|_{U} \cong U \times \mathbb{C}^{N}$, above some open subset $U \subset M$, the restriction $\left.D\right|_{U}: \Gamma\left(\left.E\right|_{U}\right) \cong$ $\mathscr{C}^{\infty}(U) \otimes \mathbb{C}^{m} \rightarrow \Gamma\left(\left.E\right|_{U}\right)$ can be written as a linear combination of compositions of operators of the form $X \otimes 1$ and $f$, with $X \in \Gamma(A)$ and $f$ a smooth endomorphism of the vector bundle $\left.E\right|_{U}$.

Proof. In a trivialization of $E$ above some open subset, we can assume that $e$ is a constant matrix.

EXAMPLE 2.19 (de Rham differential generated by $\mathscr{V}=\Gamma(A)$ ). We define for a section $\omega$ of $\Lambda^{q} A^{*}$

$$
\begin{aligned}
(d \omega)\left(X_{0}, \ldots, X_{k}\right)= & \sum_{j=0}^{q}(-1)^{j} X_{j}\left(\omega\left(X_{0}, \ldots, \hat{X}_{j}, \ldots, X_{k}\right)\right) \\
& +\sum_{0 \leq i<j \leq q}(-1)^{i+j} \omega\left(\left[X_{i}, X_{j}\right], X_{0}, \ldots, \hat{X}_{i}, \ldots, \hat{X}_{j}, \ldots, X_{k}\right) .
\end{aligned}
$$

This is well defined as $\Gamma(A)$ is closed under the Lie bracket. By choosing a local basis of $A$ we see that this defines a differential operator $\Gamma\left(\Lambda^{q} A^{*}\right) \rightarrow \Gamma\left(\Lambda^{q+1} A^{*}\right)$ generated by $\mathscr{V}=\Gamma(A)$, the de Rham differential.

Assume now that $\left.A\right|_{M_{0}}=T M_{0}$. The vector bundles $\Lambda^{q} T^{*} M_{0}$ extend to bundles $\Lambda^{q} A^{*}$ on $M$. The Cartan formula (see, e.g., [9]) says that on $M_{0}$ the de Rham differential is the de Rham differential of ordinary differential geometry.

DeFINITION 2.20. Let $E \rightarrow M$ be a vector bundle. An $A^{*}$-valued connection on $E$ is a differential operator

$$
D: \Gamma(E) \longrightarrow \Gamma\left(E \otimes A^{*}\right)
$$


such that, for any $X \in \Gamma(A)$, the induced operator $D_{X}: \Gamma(E) \rightarrow \Gamma(E)$ satisfies the usual properties of a connection:

$$
\begin{gathered}
D_{X}(f \xi)=f D_{X}(\xi)+X(f) \xi, \\
D_{f X} \xi=f D_{X}(\xi) .
\end{gathered}
$$

It is clear from (2.24a) that the operator $D_{X}$ is of first order.

Our definition of an $A^{*}$-valued connection is only slightly more restrictive than that of $A$-connection introduced in [25]. (In that paper, Evens et al. considered (2.24b) only up to homotopy.)

Clearly, if $D$ and $D^{\prime}$ are $A^{*}$-valued connections on $E$ and, respectively, $E^{\prime}$, then $D^{\prime \prime}:=$ $D \otimes 1+1 \otimes D^{\prime}$ is an $A^{*}$-valued connection on $E \otimes E^{\prime}$.

See also $[21,22]$.

3. Lie structures at infinity. In this section, we introduce the class of manifolds with a Lie structure at infinity, and we discuss some of their properties. Our definition, Definition 3.1, formalizes some definitions from [57].

In some of the first papers on the analysis on open manifolds using Lie algebras of vector fields, for example [13, 14, 16, 76], the vector fields considered were required to vanish at infinity. In order to obtain more general results, and in agreement with the more recent papers on the subject (see, e.g., $[15,57,62,77]$ ), we do not make this assumption. As a consequence, the comparison algebras that result from our setting do not have in general the property that the commutators are compact.

3.1. Definition. In the following, $\partial M$ denotes the union of all hyperfaces of a manifold with corners $M$.

DEFINITION 3.1. A Lie structure at infinity on a smooth manifold $M_{0}$ is a pair $(M, \mathscr{V})$, where

(i) $M$ is a compact manifold, possibly with corners, and $M_{0}$ is the interior of $M$;

(ii) $\mathscr{V}$ is a structural Lie algebra of vector fields;

(iii) $\varrho_{\mathscr{V}}: A_{\mathscr{V}} \rightarrow T M$ induces an isomorphism on $M_{0}$, that is, $\left.\varrho_{\mathscr{V}}\right|_{M_{0}}:\left.A_{\mathscr{V}}\right|_{M_{0}} \rightarrow T M_{0}$ is a fiberwise isomorphism.

If $M_{0}$ is compact without boundary, then it follows from the above definition that $M=$ $M_{0}$ and $A_{\mathscr{V}}=T M$, so a Lie structure at infinity on $M_{0}$ gives no additional information on $M_{0}$. The interesting cases are thus the ones when $M_{0}$ is noncompact. Note that all Examples 2.5, 2.6, 2.7, 2.8, 2.9, and 2.10 are in fact Lie structures at infinity on the interior of $M$.

Here is now an explicit test for a Lie algebra of vector fields $\mathscr{V}$ on a compact manifold with corners $M$ to define a Lie structure at infinity on the interior $M_{0}$ of $M$. This characterization of Lie structures at infinity is in the spirit of our discussion of local basis (see (2.1) and the discussion around it).

Proposition 3.2. The Lie algebra $\mathscr{V} \subset \Gamma(M ; T M)$ defines a Lie structure at infinity on $M_{0}$ if and only if the following conditions are satisfied: 
(i) $\mathscr{V} \subset \mathscr{V}_{b}$, with $\mathscr{V}_{b}$ defined in (2.3), and $\mathscr{C}^{\infty}(M) \mathscr{V} \subset \mathscr{V}$;

(ii) if $x \in M_{0}, U$ is a compact neighborhood $U$ of $x$ in $M_{0}$, and $Y$ is a vector field on $M_{0}$, then there exists $X \in \mathscr{V}$ such that $\left.X\right|_{U}=\left.Y\right|_{U}$;

(iii) if $x \in \partial M=M \backslash M_{0}$, then there are $n$ linearly independent vector fields $X_{1}, X_{2}, \ldots, X_{n}$ $\in \mathcal{V}, n=\operatorname{dim} M$, defined on a neighborhood $U$ of $x$ such that for any $X \in \mathcal{V}$, there exist smooth functions $f_{1}, \ldots, f_{n} \in \mathscr{C}^{\infty}(U)$ uniquely determined by

$$
X=\sum_{k=1}^{n} f_{k} X_{k} \quad \text { on } U \text {; }
$$

(iv) there are functions $f_{i j k} \in \mathscr{C}^{\infty}(U)$ (in particular smooth on the boundary $\partial M \cap U$ ) such that the vector fields $X_{j}$ from (iii) satisfy $\left[X_{i}, X_{j}\right]=\sum_{k=1}^{n} f_{i j k} X_{k}$ on $U$.

Proof. The proof is an immediate translation of the definition of a manifold with a Lie structure at infinity using the description of projective $\mathscr{C}^{\infty}(M)$-module given at the end of Section 2.1 (especially (2.1)).

3.2. Riemannian manifolds with Lie structures at infinity. We now consider Riemannian metrics on $A \rightarrow M$.

DEFINITION 3.3. A manifold $M_{0}$ with a Lie structure at infinity $(M, \mathscr{V})$, together with a Riemannian metric on $A=A_{\mathscr{V}}$, that is, a smooth positive definite symmetric 2-tensor $g$ on $A$, is called a Riemannian manifold $M_{0}$ with a Lie structure at infinity.

In particular $g$ defines a Riemannian metric on $M_{0}$. The geometry of these metrics will be the topic of the next section. Note that the metrics on $M_{0}$ that we obtain are not restrictions of Riemannian metrics on $M$. In the following section, we will prove for example that $\left(M_{0}, g\right)$ is a complete Riemannian metric. Any curve joining a point on the boundary $\partial M$ to the interior $M_{0}$ is necessarily of infinite length.

EXAMPLE 3.4 (manifolds with cylindrical ends). A manifold $M$ with cylindrical ends is obtained by attaching to a manifold $M_{1}$ with boundary $\partial M_{1}$ the cylinder $(-\infty, 0] \times \partial M_{1}$, using a tubular neighborhood of $\partial M_{1}$, where the metric is assumed to be a product metric. The metric on the cylinder is also assumed to be the product metric. Let $t$ be the coordinate of $(-\infty, 0]$. By the change of variables $x=e^{t}$, we obtain that $M$ is diffeomorphic to the interior of $M_{1}$ and $\mathscr{V}=\mathscr{V}_{b}$. Other changes of variables lead us to different Lie structures at infinity. Similarly, products of manifolds with cylindrical ends can be modeled by manifolds with corners and the structural Lie algebra of vector fields $\mathscr{V}_{b}$. This applies also to manifolds that are only locally at infinity products of manifolds with cylindrical ends.

3.3. Bi-Lipschitz equivalence. It turns out that the metric on a manifold with a Lie structure at infinity is essentially unique, namely, any two such metrics are bi-Lipschitz equivalent (see the corollary below).

LEMMA 3.5. Assume that a manifold $M_{0}$ which is the interior of a compact manifold with corners $M$ carries two Lie structures at infinity, $\left(M, \mathscr{V}_{1}\right)$, and $\left(M, \mathscr{V}_{2}\right)$, satisfying $\mathscr{V}_{1} \subset \mathscr{V}_{2}$. Furthermore, let $g_{j}$ be Riemannian metrics on $A_{\mathscr{V}_{j}}, j=1,2$. Then there is a 
constant $C$ such that

$$
g_{2}(X, X) \leq C g_{1}(X, X) \quad \forall X \in T M_{0}
$$

Proof. The pullback of $g_{2}$ to $A_{V_{1}}$ is a nonnegative symmetric two-tensor on $A_{V_{1}}$. The statement then follows from the compactness of $M$.

As a consequence, the volume element of $g_{2}$ is bounded by a multiple of the volume element of $g_{1}$. Furthermore, we have inclusions of $L^{p}$-functions: $L^{p}\left(M_{0}, g_{1}\right) \hookrightarrow$ $L^{p}\left(M_{0}, g_{2}\right)$.

COROLLARY 3.6. If two Riemannian metrics $g_{1}, g_{2}$ on $M_{0}$ are Riemannian metrics for the same Lie structure at infinity $(M, \mathscr{V})$, then they are bi-Lipschitz, that is, there is a constant $C>0$ with

$$
C^{-1} g_{2}(X, X) \leq g_{1}(X, X) \leq C g_{2}(X, X)
$$

for all $X \in T M_{0}$. In particular, $C^{-1} d_{2}(x, y) \leq d_{1}(x, y) \leq C d_{2}(x, y)$, where $d_{i}$ is the metric on $M_{0}$ associated to $g_{i}$.

Proof. The first part is clear. The proof of the last statement is obtained by comparing the metrics on a geodesic for one of the two metrics.

4. Geometry of Riemannian manifolds with Lie structures at infinity. We now discuss some geometric properties of Riemannian manifolds with a Lie structure at infinity. We begin with a simple observation about volumes.

4.1. Volume. Let $d$ vol be the volume element on $M_{0}$

Proposition 4.1. Let $M_{0}$ be a Riemannian manifold with Lie structure $(M, \mathscr{V}, g)$ at infinity. Let $f \geq 0$ be a smooth function on $M$. If $\int_{M_{0}} f d \mathrm{vol}<\infty$, then $f$ vanishes on each boundary hyperface of $M$. In particular, the volume of any noncompact Riemannian manifold with a Lie structure at infinity is infinite.

Proof. Because of Lemma 3.5, we can assume that $\Gamma(A)$ are the vector fields tangential to the boundary. For simplicity in notation, we assume that $M$ is a compact manifold with boundary. Let $d \mathrm{vol}^{\prime}$ be the volume element on $M$ associated to some metric on $M$ that is smooth up to the boundary. Then $d \mathrm{vol} \geq C x^{-1} d \mathrm{vol}^{\prime}$ for any boundary defining function $x$ and a constant $C$.

So, if $f$ is nonzero on $\partial M$ with defining function $x$, then

$$
\int_{M_{0}} f d \mathrm{vol} \geq \int_{M} f x^{-1} d \operatorname{vol}^{\prime}=\infty
$$

4.2. Connections and curvature. Most of the natural differential operators between bundles functorially associated to the tangent bundle extend to differential operators generated by $\mathscr{V}$, with the tangent bundle replaced by $A$. The main example is the LeviCivita connection. 
LEMMA 4.2. Let $M_{0}$ be a Riemannian manifold with a Lie structure $(M, \mathscr{V}, g)$ at infinity. Then the Levi-Civita connection $\nabla: \Gamma\left(T M_{0}\right) \rightarrow \Gamma\left(T M_{0} \otimes T^{*} M_{0}\right)$ extends to a differential operator in Diff ( $\left.\mathscr{V} ; A, A \otimes A^{*}\right)$, also denoted by $\nabla$. In particular, $\nabla_{X} \in \operatorname{Diff}(\mathscr{V} ; A)$, for any $X \in \Gamma(A)$, and it satisfies

$$
\nabla_{X}(f Y)=X(f) Y+f \nabla_{X}(Y), \quad X\langle Y, Z\rangle=\left\langle\nabla_{X} Y, Z\right\rangle+\left\langle Y, \nabla_{X} Z\right\rangle
$$

for all $X, Y, Z \in \Gamma(A)$ and $f \in \mathscr{C}^{\infty}(M)$. Moreover, the above equations uniquely determine $\nabla$.

Proof. Suppose $X, Y \in \mathscr{V}=\Gamma(A) \subset \Gamma(T M)$. We will define $\nabla_{X} Y$ on $M_{0}$ using the usual Levi-Civita connection $\nabla$ on $T M_{0}$. We need to prove that there exists $X_{1} \in \Gamma(A)$ whose restriction to $M_{0}$ is $\nabla_{X} Y$.

Recall (e.g., from [9]) that the formula for $\nabla_{X} Y$ is given by

$$
\begin{aligned}
2\left\langle\nabla_{X} Y, Z\right\rangle= & \langle[X, Y], Z\rangle-\langle[Y, Z], X\rangle+\langle[Z, X], Y\rangle \\
& +X\langle Y, Z\rangle+Y\langle Z, X\rangle-Z\langle X, Y\rangle .
\end{aligned}
$$

Suppose $X, Y, Z \in \Gamma(A)$ in the above formula. We see then that the function $2\left\langle\nabla_{X} Y, Z\right\rangle$, which is defined a priori only on $M_{0}$, extends to a smooth function on $M$. Since the inner product $\langle\cdot, \cdot\rangle$ is the same on $A$ and on $T M_{0}$ (where they are both defined), we see that the above equation determines $\nabla_{X} Y$ as a smooth section of $A$. This completes the proof.

The above lemma has interesting consequences about the geometry of Riemannian manifolds with Lie structures at infinity.

Using the terminology of $A^{*}$-valued connections (see Definition 2.20), Lemma $4.2 \mathrm{can}$ be formulated as saying that the usual Levi-Civita connection on $M_{0}$ extends to an $A^{*}$ valued connection on $A$. Similarly, we get $A^{*}$-valued connections on $A^{*}$ and on all vector bundles obtained functorially from $A$. We use this remark to obtain a canonical $A^{*}$ valued connection on the bundles $A^{* \otimes k} \otimes \Lambda^{2} A^{*} \otimes \operatorname{End}(A)$. (Here $E^{\otimes k}$ denotes $E \otimes \cdots \otimes E$, $k$-times, as usual.)

We define the Riemannian curvature tensor as usual:

$$
R(X, Y):=\nabla_{X} \nabla_{Y}-\nabla_{Y} \nabla_{X}-\nabla_{[X, Y]} \in \Gamma\left(\operatorname{End}\left(T M_{0}\right)\right)
$$

where $X, Y \in \Gamma\left(T M_{0}\right)$. We will regard $R$ as a section of $\Lambda^{2} T^{*} M_{0} \otimes \operatorname{End}\left(T M_{0}\right)$. Then the covariant derivatives $\nabla^{k} R \in \Gamma\left(T^{*} M_{0}^{\otimes k} \otimes \Lambda^{2} T^{*} M_{0} \otimes \operatorname{End}\left(T M_{0}\right)\right)$ are defined.

COROLLARY 4.3. If $M_{0}$ and $\nabla$ are as above (Lemma 4.2), then the Riemannian curvature tensor $R(X, Y)$ extends to an endomorphism of $A$, for all $X, Y \in \Gamma(A)$. Moreover, each covariant derivative $\nabla^{k} R$ extends to a section of $A^{* \otimes k} \otimes \Lambda^{2} A^{*} \otimes \operatorname{End}(A)$ over $M$ and hence they are all bounded on $M_{0}$.

Proof. Fix $X, Y \in \Gamma(A)$. Then $[X, Y] \in \Gamma(A)$, and hence

$$
\nabla_{X}, \nabla_{Y}, \nabla_{[X, Y]} \in \operatorname{Diff}(\mathscr{V} ; A)
$$


by Lemma 4.2. It follows that $R(X, Y) \in \operatorname{Diff}(\mathscr{V} ; A)$. Since $R(X, Y)$ induces an endomorphism of $T M_{0}$ and $M_{0}$ is dense in $M$, it follows that $R(X, Y) \in \operatorname{End}(A)$.

Once we have obtained that $R \in \Gamma\left(\Lambda^{2} A^{*} \otimes \operatorname{End}(A)\right)$, we can apply the $A^{*}$-valued LeviCivita connection to obtain

$$
\nabla^{k} R \in \Gamma\left(A^{* \otimes k} \otimes \Lambda^{2} A^{*} \otimes \operatorname{End}(A)\right) .
$$

The boundedness of $\nabla^{k} R$ follows from the fact that $M$ is compact.

The covariant derivative

$$
\nabla_{X}: \Gamma\left(A^{\otimes k} \otimes A^{* \otimes j}\right) \longrightarrow \Gamma\left(A^{\otimes k} \otimes A^{* \otimes(j+1)}\right), \quad X \in \Gamma(A),
$$

will be called, by abuse of notation, the $A^{*}$-valued Levi-Civita connection, for any $k$ and $j$. Sometimes, when no confusion can arise, we will simply call this $A^{*}$-valued connection $\nabla$ the Levi-Civita connection.

\subsection{Ehresmann connections}

DEFINITION 4.4. Let $\pi_{N}: N \rightarrow M$ be a submersion of manifolds with corners, and let $A \rightarrow M$ be a boundary tangential Lie algebroid. Smooth sections of the bundle $\wedge^{p} \pi_{N}^{\#} A \rightarrow N$ are called $A^{*}$-valued $p$-forms on $N$.

The fiber of $A$ in $p \in M$ is denoted by $A_{p}$.

DEFINITION 4.5. An Ehresmann connection on $\pi_{N}: N \rightarrow M$ with respect to $A$ is a smooth field $x \mapsto \tau_{x}, x \in N$, of $n$-dimensional subspaces of $\pi_{N}{ }^{\#} A$ such that $\left(\pi_{N}\right)_{*}$ : $\pi_{N}{ }^{\#} A \rightarrow A$ restricts to an isomorphism $\tau_{x} \rightarrow A_{\pi_{N} x}$, for all $x \in N$, the inverse $\left(\left(\pi_{N}\right)_{*}\right)^{-1}$ : $A_{\pi_{N} x} \rightarrow \tau_{x}$ is called a horizontal lift.

We chose the terminology "Ehresmann connection" to honor the important work of Ehresmann on the subject [19].

EXAMPLE 4.6. (a) Let $\pi: V \rightarrow M$ be a vector bundle. The definition of an Ehresmann connection generalizes the notion of $A^{*}$-valued connection (in the sense of vector bundle connections). In fact, let $\nabla$ be an $A^{*}$-valued connection. Then we obtain the Ehresmann connection as follows: for $X_{0} \in V, p=\pi\left(X_{0}\right)$, we extend $X_{0}$ to a local section $X: U \rightarrow V$, where $U$ is a neighborhood of $p$ in $M$. We define the horizontal lift

$$
H_{X_{0}}: A_{p} \longrightarrow\left(\pi^{\#} A\right)_{X_{0}}, \quad Y \longmapsto\left(X_{*}\right)_{p}(Y)-\nabla_{Y} X,
$$

where $\nabla_{Y} X \in V_{p} \subset\left(\pi^{\#} A\right)_{X_{0}}$. It is easy to check that this map does not depend on the extension $X$ of $X_{0}$, that $H_{X_{0}}$ is injective, and that we have $\left(\pi_{*}\right)_{X_{0}} \circ H_{X_{0}}=$ id.

Then $\tau_{X_{0}}:=i m H_{X_{0}}$ is an Ehresmann connection on $V$. The associated Ehresmann connection completely characterizes the $A^{*}$-valued connection. However, there are Ehresmann connections on $V$ that do not come from $A^{*}$-valued connections (they are not "compatible" with the vector space structure).

(b) If the $A^{*}$-valued connection is metric with respect to a chosen metric on $V$, then the Ehresmann connection is tangential to the sphere bundle in $V$ with respect to that metric. 
4.4. Geodesic flow. For any boundary tangential Lie algebroid $A$ equipped with a metric, let $S(A)$ be the unit tangent sphere in $A$,

$$
S(A):=\{v \in A \mid\|v\|=1\}
$$

The canonical projection map $\pi: S(A) \rightarrow M$ is a submersion of manifolds with corners. Let $\pi^{\#} A$ be the thick pullback of $A$.

The manifold (with corners) $S(A)$ carries an Ehresmann connection and a horizontal lift $H$ given by the Levi-Civita connection on $A$.

Definition 4.7. The geodesic spray is defined to be the map

$$
S(A) \ni X \longmapsto H_{X}(X) \in \pi^{\#} A,
$$

which defines a section $S$ of $\pi^{\#} A \rightarrow S(A)$. The flow of this vector field is called the geodesic flow.

Restricted to the interior of the manifold, these concepts recover the analogous concepts of ordinary Riemannian geometry.

By definition, the image of $S$ through the anchor map is a vector field along $S(A)$ that is tangential to all the boundary faces of $S(A)$. These boundary faces are preimages of the boundary faces of $M$ under $\pi$.

LEMMA 4.8. Let $A$ be a boundary tangential Lie algebroid and let $X \in \Gamma(A)$. Then $X$ is complete in the sense that the flow lines $\varphi_{t}$ of $X$ are defined on $\mathbb{R}$. The flow $\varphi_{t}$ preserves the boundary depth. In particular, flow lines emanating from $N_{0}:=N \backslash \partial N$ stay in $N_{0}$.

Proof. For any boundary defining function $x_{H}$, one has

$$
\left.\frac{d}{d t}\right|_{t=0} x_{H}\left(\varphi_{t}\right)=d x_{H}(X)=0
$$

hence the flow preserves the boundary depth. In particular, the flow preserves the boundary. Let $I=(a, b)$ be a maximal open interval on which one particular flow line is defined. Let $t_{i} \rightarrow b$. Assume that $b<\infty$. Since $N$ is compact, after passing to a suitable subsequence, we can assume that $\varphi_{t_{i}}$ converges to $p \in N$. In a neighborhood of $p$, the flow exists, which contradicts the maximality of $b$. Hence $b=\infty$. The proof for $a=-\infty$ is similar.

By applying this lemma to the geodesic flow on $N=S(A)$, we obtain two corollaries.

COROLlary 4.9. Let $M_{0}$ be a Riemannian manifold with a Lie structure $(M, \mathscr{V}, g)$ at infinity. Then $M_{0}$ is complete in the induced metric $g$.

COROLLARY 4.10. Let $M_{0}$ be a Riemannian manifold with a Lie structure $(M, \mathscr{V}, g)$ at infinity. Let $X \in A_{p}, p \in M$. Then the boundary depth of $\exp _{p} A_{p}$ equals the boundary depth of $p$. 
4.5. Positive injectivity radius. We already know that Riemannian manifolds with a Lie structure at infinity are complete and have bounded sectional curvature. For many analytic statements, it is very helpful if we also know that the injectivity radius $\operatorname{inj}\left(M_{0}, g\right)=\inf _{p \in M_{0}} \operatorname{inj}_{p}$ is positive. For example, see [39], where a "uniform bounded calculus of pseudodifferential operators" was defined on a manifold with bounded geometry. Hebey [29, Corollary 3.19] proved Sobolev embeddings for manifolds with bounded geometry, that is, complete Riemannian manifolds with positive injectivity radius and bounded covariant derivatives $\nabla^{k} R$ of the Riemannian curvature tensor $R$. We will say more about this in a future paper.

CONJECTURE 4.11. Any Riemannian manifold with a Lie structure at infinity has positive injectivity radius.

We now introduce two conditions on a Riemannian manifold $M_{0}$ with a Lie structure at infinity $(M, \mathscr{V})$, (see Definitions 4.12 and 4.16), and prove that if any of these conditions holds, then the injectivity radius of $M_{0}$ is positive.

DEFINITION 4.12. A manifold $M_{0}$ with a Lie structure at infinity $(M, \mathscr{V})$ is said to satisfy the local closed extension property for 1 -forms if any $p \in \partial M$ has a small neighborhood $U \subset M$ such that any $\alpha_{p} \in A_{p}^{*}$ extends to a closed 1-form on $U$.

EXAMPLE 4.13. For the $b$-calculus, the local closed extension property holds because in the notation of Example 2.5, the locally defined closed 1-forms $d x^{j} / x^{j}$ and $d y^{k}$ span $A_{p}^{*}=\left(T_{p}^{b} M\right)^{*}$ for any $p \in \partial M$.

THEOREM 4.14. Let $M_{0}$ be a manifold with a Lie structure at infinity $(M, \mathscr{V})$ which satisfies the local closed extension property. Then for any Riemannian metric $g$ on $A$, the injectivity radius of $\left(M_{0}, g\right)$ is positive.

Proof. We prove the theorem by contradiction. If the injectivity radius is zero, then, as the curvature is bounded, there is a sequence of geodesics loops $c_{i}:\left[0, a_{i}\right] \rightarrow M_{0}$, parametrized by arc-length, with $a_{i} \rightarrow 0$. Because of the compactness of $S(A)$, we can choose a subsequence such that $\dot{c}_{i}(0)$ converges to a vector $v \in S(A)$. Obviously, the basepoint of $v$ has to be in $\partial M$.

By the local closed extension property, there is a closed 1-form $\alpha$ on a small neighborhood of the basepoint of $v$ such that $\alpha(v) \neq 0$. On the other hand, because of the closedness of $\alpha$, we get, for sufficiently large $i$,

$$
0=\int_{0}^{1} \alpha\left(\dot{c}_{i}\left(a_{i} t\right)\right) d t=\int_{0}^{1} \alpha\left(\varphi_{a_{i} t}\left(\dot{c}_{i}(0)\right)\right) d t
$$

where $\varphi_{t}: S(A) \rightarrow S(A)$ denotes the geodesic flow. As $i \rightarrow \infty$, the integrand converges uniformly to $\alpha(v)$, thus we obtain the contradiction $\alpha(v)=0$.

In the remainder of this subsection, we will prove another sufficient criterion.

DEFINITION 4.15. Let $\varphi:[0, \infty)^{n-k} \times \mathbb{R}^{k} \rightarrow U \subset M$ be a local parametrization of $M$, that is, $\varphi^{-1}$ is a coordinate chart. Then, for $v \in \mathbb{R}^{n}$, the local vector field $\varphi_{*}(v)$, that is, 
the image of a constant vector field $v$ on $\mathbb{R}^{n}$, is called a coordinate vector field with respect to $\varphi$.

DEFINITION 4.16. A manifold $M_{0}$ with a Lie structure at infinity $(M, \mathscr{V})$ is said to satisfy the coordinate vector field extension property if $A_{\mathscr{V}}$ carries a Riemannian metric $g$ such that, for any $p \in \partial M$, there is a parametrization $\varphi:[0, \infty)^{n-k} \times \mathbb{R}^{k} \rightarrow U$ of a neighborhood $U$ of $p$ such that

(i) for any $v \in \mathbb{R}^{n} \backslash\{0\}$, the normalized coordinate vector field

$$
X_{v}=\frac{\varphi_{*}(v)}{\sqrt{g\left(\varphi_{*}(v), \varphi_{*}(v)\right)}},
$$

which a priori is only defined on $U \cap M_{0}$, extends to a section of $\left.A\right|_{U}$;

(ii) for linearly independent vectors $v$ and $w, X_{v}(p)$ and $X_{w}(p)$ are linearly independent.

Note that property (i) is equivalent to claiming that

$$
f_{v}:=\frac{1}{\sqrt{g\left(\varphi_{*}(v), \varphi_{*}(v)\right)}}
$$

extends to a smooth function on $M$.

THEOREM 4.17. Let $M_{0}$ be a manifold with a Lie structure at infinity $(M, \mathscr{V})$ that satisfies the coordinate vector field extension property. Then, for any Riemannian metric $g$ on $A$, the injectivity radius of $\left(M_{0}, g\right)$ is positive.

The theorem will follow right away from Proposition 4.19 and Lemmas 4.23 and 4.24, which we proceed to state and prove after the following definition.

DEFINITION 4.18. For $C \geq 1$ and $\varepsilon>0,\left(M_{0}, g\right)$ is said to be locally $C$-bi-Lipschitz to an $\varepsilon$-ball if each point $p \in M_{0}$ has a neighborhood that is bi-Lipschitz diffeomorphic to a flat ball of radius $\varepsilon$ with bi-Lipschitz constant $C$.

Proposition 4.19. Let $\left(M_{0}, g\right)$ be a complete Riemannian manifold with bounded sectional curvature. Then the following conditions are equivalent:

(1) $\left(M_{0}, g\right)$ has positive injectivity radius;

(2) there are numbers $\delta_{1}>0$ and $C>0$ such that any loop of length $\delta \leq \delta_{1}$ is the boundary of a disk of diameter less than or equal to $C \cdot \delta$;

(3) there are $C>0$ and $\varepsilon>0$ such that $\left(M_{0}, g\right)$ is locally $C$-bi-Lipschitz to a ball of radius $\varepsilon$.

Proof. $(1) \Rightarrow(3)$. Let $\varrho>0$ be the injectivity radius of $\left(M_{0}, g\right)$. Then $B_{\varrho / 2}(p)$ is $C$-biLipschitz to a flat ball with $C$ independent from $p$.

$(3) \Rightarrow(2)$. Under the condition of (3), any loop of length less than or equal to $2 \varepsilon / C$ with basepoint $p$ lies completely inside $B_{\varepsilon / C}(p)$. On the other hand, $B_{\varepsilon / C}(p)$ is contained in a neighborhood $U$ of $p$ which is $C$-bi-Lipschitz to a flat ball of radius $\varepsilon$. Inside a flat ball, any short loop is the boundary of a disk of small diameter. Hence, (2) follows from (3) with $\delta_{1}:=2 \varepsilon / C$ and with the same $C$ in (2) as in (3). 
$(2) \Rightarrow(1)$. Because sectional curvature is bounded from above, there is a $\varrho>0$ such that there are no conjugate points along curves of length smaller than $\varrho$. For each $p \in M$, the exponential map is a local diffeomorphism from $B_{\varrho}(p)$ into $M$. We want to show that the exponential map is injective on any ball of radius $\varepsilon:=\min \left\{\delta_{1} / 2, \varrho /(4 C)\right\}$. For this we assume that $\exp _{p}\left(q_{1}\right)=\exp _{p}\left(q_{2}\right), q_{1}, q_{2} \in B_{\varepsilon}(0) \subset T_{p} M$. Then

$$
t \longmapsto \begin{cases}\exp _{p}\left(t q_{1}\right), & \text { for } 0 \leq t \leq 1 \\ \exp _{p}\left((2-t) q_{2}\right), & \text { for } 1 \leq t \leq 2\end{cases}
$$

is a closed loop of length less than or equal to $2 \varepsilon$. Because of the conditions in the proposition, this loop is the boundary of a disk of diameter less than or equal to $\varrho / 2$. Because the exponential map is a local diffeomorphism, it is not difficult to see that such disks lift to $T_{p} M$. Hence, $q_{1}=q_{2}$, which yields injectivity.

As a consequence of this proposition, the property of having positive injectivity radius is a bi-Lipschitz invariant inside the class of complete Riemannian manifolds with bounded curvature.

COROLlary 4.20. Let $g_{1}$ and $g_{2}$ be two complete metrics on $M_{0}$ such that there is $C>0$ with

$$
\begin{gathered}
C^{-1} g_{1} \leq g_{2} \leq C g_{1}, \\
\left|R_{g_{1}}\right|<C, \quad\left|R_{g_{2}}\right|<C .
\end{gathered}
$$

Then $\left(M_{0}, g_{1}\right)$ has positive injectivity radius if and only if $\left(M_{0}, g_{2}\right)$ has positive injectivity radius.

Proof. Proposition 4.19 gives necessary and sufficient criteria for positive injectivity radius, that are bi-Lipschitz equivalent.

Together with Corollary 3.6 we obtain the following corollary.

COROLLARY 4.21. Suppose that $M_{0}$ is a manifold with a Lie structure $(M, \mathcal{V})$ at infinity and let $g$ and $h$ be two metrics on $A$. Then $\left(M_{0},\left.g\right|_{M_{0}}\right)$ has positive injectivity radius if and only if $\left(M_{0},\left.h\right|_{M_{0}}\right)$ has positive injectivity radius.

DEFINITION 4.22. Let $M_{0}$ be a Riemannian manifold with a Lie structure $(M, \mathscr{V}, g)$ at infinity. We say that the Lie structure at infinity is controlled if for all $p \in \partial M$, there are a parametrization $\varphi:[0, \infty)^{n-k} \times \mathbb{R}^{k} \rightarrow U$ around $p$, a $\delta>0$, and a constant $C>0$ such that, for all $x \in M_{0} \cap U$ and all $v \in \mathbb{R}^{n}$, the inequality

$$
\max _{y \in B_{\delta}(x)} g_{y}\left(\varphi_{*}(v)_{y}, \varphi_{*}(v)_{y}\right)<C \min _{y \in B_{\delta}(x)} g_{y}\left(\varphi_{*}(v)_{y}, \varphi_{*}(v)_{y}\right)
$$

holds. Here $B_{\delta}(x)$ denotes the ball of radius $\delta$ around $x$ with respect to the metric $g$.

LEMMA 4.23. Let $M_{0}$ be a manifold with a Lie structure at infinity $(M, \mathscr{V})$ satisfying the coordinate vector field extension property with the metric $g$. Then $(M, \mathscr{V}, g)$ is controlled. 
Proof. Let $\varphi$ be a parametrization of a neighborhood $U$ of $p \in \partial M$ and let $\varphi_{*}(v)$ and $\varphi_{*}(w)$ be arbitrary coordinate vector fields. Because of Property (i) in Definition 4.16, the (local) functions $f_{v}:=1 / \sqrt{g\left(\varphi_{*}(v), \varphi_{*}(v)\right)}$ and $f_{w}:=1 / \sqrt{g\left(\varphi_{*}(w), \varphi_{*}(w)\right)}$ extend to the boundary, and $X_{v}:=f_{v} \varphi_{*}(v)$ and $X_{w}:=f_{w} \varphi_{*}(w)$ are sections of $A$. For linearly independent $v$ and $w$, we calculate

$$
\begin{aligned}
{\left[X_{v}, X_{w}\right] } & =\left[f_{v} \varphi_{*}(v), f_{w} \varphi_{*}(w)\right] \\
& =X_{v}\left(f_{w}\right) \varphi_{*}(w)-X_{w}\left(f_{v}\right) \varphi_{*}(v) \\
& =X_{v}\left(\log f_{w}\right) X_{w}-X_{w}\left(\log f_{v}\right) X_{v}
\end{aligned}
$$

This is again a section of $A$, and because of property (ii) in Definition $4.16, X_{v}$ and $X_{w}$ are even linearly independent of the boundary $\partial M$. As a consequence, $X_{v}\left(\log f_{w}\right)$ is bounded. On the other hand, if $v=w$, then because $f_{w}$ extends to the boundary, $X_{v}\left(\log f_{w}\right)=f_{v} \varphi_{*}(v)\left(f_{v}\right) / f_{v}=\varphi_{*}(v)\left(f_{v}\right)$ is also bounded. Hence, in both cases,

$$
\left|X_{v}\left(\log g\left(\varphi_{*}(w), \varphi_{*}(w)\right)\right)\right| \leq C .
$$

By summing up, one immediately sees that $C$ can be chosen independently from the choice of $v$. Hence, for fixed $w,(4.19)$ holds uniformly for any unit vector $X$.

We take two arbitrary points $y_{1}, y_{2} \in B_{\delta}(x), x \in U \cap M_{0}$. We can assume that $B_{\delta}(x) \subset$ $U$. We join $y_{1}$ and $y_{2}$ by a path $c:[0, \tilde{\delta}] \rightarrow M_{0}$, parametrized by arc-length, $\delta \leq 2 \delta$. We estimate

$$
\begin{aligned}
& \left|\log \left(\frac{g_{c(0)}\left(\varphi_{*}(w)_{c(0)}, \varphi_{*}(w)_{c(0)}\right)}{g_{c(\tilde{\delta})}\left(\varphi_{*}(w)_{c(\tilde{\delta})}, \varphi_{*}(w)_{c(\tilde{\delta})}\right)}\right)\right| \\
& \quad=\left|\int_{0}^{\tilde{\delta}} \partial_{\dot{\mathcal{c}}(t)}\left(\log g_{c(t)}\left(\varphi_{*}(w)_{c(t)}, \varphi_{*}(w)_{c(t)}\right)\right) d t\right| \leq \tilde{\delta} C .
\end{aligned}
$$

Hence, the quotient is bounded by an expression that depends only on $p, U, \delta$, and global data. Thus, $(M, \mathscr{V}, g)$ is controlled.

LEMMA 4.24. If the boundary tangential Lie algebroid is controlled, then there are $C>0$ and $\varepsilon>0$ such that $\left(M_{0}, g\right)$ is locally $C$-bi-Lipschitz to a $\varepsilon$-ball.

Proof. On the ball $B_{\delta}(x)$, we regard two metrics: the original metric $g$ and the metric $\tilde{g}$ with $g_{x}=\tilde{g}_{x}$, and $\tilde{g}_{x}$ is constant in the local coordinate chart.

These metrics are bi-Lipschitz on $B$ with a bi-Lipschitz constant $C_{1}$. Thus, the ball $\tilde{B}_{x}$ of radius $\delta / C_{1}$ around $x$ with respect to the metric $\tilde{g}$ is a flat ball. Hence, $\left(\tilde{B}_{x}\right)_{x \in M_{0}}$ are neighborhoods that are uniformly bi-Lipschitz to $\delta / C_{1}$-balls. This completes the proof of Theorem 4.17 .

We continue with some examples and applications.

EXAMPLE 4.25. Examples 2.5-2.10 satisfy the coordinate vector field extension property and hence have positive injectivity radius. 
EXAMPLE 4.26. Let $M=[0, \infty) \times \mathbb{R} \times \mathbb{R} \ni(t, x, y)$. The vector fields $X=t^{2} \partial_{t}, Y=$ $e^{-C / t} \partial_{x}$, and $Z=e^{-C / t} \partial_{y}$ span a free $C^{\infty}(M)$-module that is closed under Lie brackets. Hence, it is a structural Lie algebra of vector fields $\mathscr{V}$. We define a metric $g$ by claiming that $X, Y$, and $Z$ are orthonormal, then $(M, g, \mathscr{V})$ satisfies the coordinate vector field extension property.

EXAMPLE 4.27. Let $M=[0, \infty) \times \mathbb{R} \times \mathbb{R} \ni(t, x, y)$. The vector fields $X=t^{2} \partial_{t}, Y=$ $e^{-C / t}\left(\sin (1 / t) \partial_{x}+\cos (1 / t) \partial_{y}\right)$, and $Z=e^{-C / t}\left(\cos (1 / t) \partial_{x}-\sin (1 / t) \partial_{y}\right)$ span a free $C^{\infty}(M)$-module that is closed under Lie brackets. Hence it is a structural Lie algebra of vector fields $\mathscr{V}$. We define a metric by claiming that $X, Y$, and $Z$ are orthonormal. In this example, the normalized coordinate vector fields $e^{-C / t} \partial_{x}$ and $e^{-C / t} \partial_{y}$ are not contained in $\mathscr{V}$. Hence $(M, \mathscr{V})$ does not satisfy the coordinate vector field extension property. However, $\left(M_{0}:=M \backslash \partial M, g\right)$ is isometric to the interior of the previous example. Corollary 4.20 together with Theorem 4.17 will show that $\left(M_{0}, g\right)$ has positive injectivity radius, although the conditions in Theorem 4.17 are not satisfied for $(M, \mathscr{V})$ directly.

4.6. Adjoints of differential operators. In what follows, we will fix a metric on $A$, thus we obtain a Riemannian manifold $\left(M_{0}, g\right)$ with a Lie structure at infinity, which will remain fixed throughout this section.

We now discuss adjoints of operators in Diff $(\mathscr{V})$. The metric on $M_{0}$ defines a natural volume element $\mu$ on $M_{0}$, and hence it defines also a Hilbert space $L^{2}\left(M_{0}, d \mu\right)$ with inner product $\left(g_{1}, g_{2}\right):=\int_{M_{0}} g_{1} \overline{g_{2}} d \mu$. The formal adjoint $D^{\#}$ of a differential operator $D$ is then defined by the formula

$$
\left(D g_{1}, g_{2}\right)=\left(g_{1}, D^{\sharp} g_{2}\right) \quad \forall g_{1}, g_{2} \in \mathscr{C}_{c}^{\infty}\left(M_{0}\right)
$$

We would like to prove that $D^{\sharp} \in \operatorname{Diff}(\mathscr{V})$ provided that $D \in \operatorname{Diff} \mathscr{V}$. To check this, we first need a lemma. Fix a local orthonormal basis $X_{1}, \ldots, X_{n}$ of $A$ (on some open subset of $M)$. Then $\nabla_{X_{i}} X=\sum c_{i j}(X) X_{j}$ for some smooth functions $c_{i j}(X)$. Then $\operatorname{div}(X):=$ $-\sum c_{j j}(X)$ is well defined and gives rise to a smooth function on $M$. See [27, Chapter IV.A].

LEMMA 4.28. Let $X \in \Gamma(A)$ and $f \in \mathscr{C}_{c}^{\infty}\left(M_{0}\right)$. Then

$$
\int_{M_{0}} X(f) \mu=\int_{M_{0}} f \operatorname{div}(X) \mu
$$

In particular, the formal adjoint of $X$ is $X^{\sharp}=-X+\operatorname{div}(X) \in \operatorname{Diff}(\mathscr{V})$.

Proof. We know (see [27, Example 4.6]) that

$$
\operatorname{div}(f X)=f \operatorname{div}(X)-X(f)
$$

The divergence theorem (see, e.g., [27, Chapter IV.A]) states for $X \in \Gamma(A)$ and compactly supported functions $f$ that 


$$
0=\int_{M_{0}} \operatorname{div}(f X) \mu=\int_{M_{0}} f \operatorname{div}(X) \mu-\int_{M_{0}} X(f) \mu
$$

so

$$
\int_{M_{0}} X(f) \mu=\int_{M_{0}} f \operatorname{div}(X) \mu
$$

Now, if we set $f=g_{1} \overline{g_{2}}$, we see directly that

$$
\int_{M_{0}} g_{1} \overline{X\left(g_{2}\right)} \mu+\int_{M_{0}} X\left(g_{1}\right) \overline{g_{2}} \mu=\int_{M_{0}} X\left(g_{1} \overline{g_{2}}\right) \mu=\int_{M_{0}} \operatorname{div}(X) g_{1} \overline{g_{2}} \mu .
$$

This implies the formula for the adjoint of $X$.

COROLLARY 4.29. Let $\left(M_{0}, g\right)$ be a Riemannian manifold with a Lie structure at infinity. The algebra Diff $(\mathscr{V})$ is closed under taking formal adjoints. Similarly, if $E$ is a Hermitian vector bundle on $M$, then Diff $(\mathscr{V} ; E)$ is also closed under taking formal adjoints.

Proof. The formal adjoint of a vector field $X \in \mathscr{V}$, when regarded as a differential operator on $M_{0}$, is given by $X^{\sharp}=-X+\operatorname{div}(X)$. The adjoint of $f \in \mathscr{C}^{\infty}(M)$ is given by $f^{\sharp}=\bar{f}$. Since Diff $(\mathscr{V})$ is generated as an algebra by operators of the form $X$ and $f$, with $X$ and $f$ as above, and $\left(D_{1} D_{2}\right)^{\#}=D_{2}^{\#} D_{1}^{\#}$, this proves that Diff ( $\left.\mathscr{V}\right)$ is closed under taking adjoints.

If $E$ is a Hermitian vector bundle, then we can choose the embedding $E \rightarrow M \times \mathscr{C}^{N}$ to preserve the metric. Then the projection $e$ onto the range of $E$ is a selfadjoint projection in $M_{N}(\operatorname{Diff}(\mathscr{V}))$. The equation $e^{*}=e$ satisfied by $e$ guarantees that $\operatorname{Diff}(\mathscr{V} ; E):=$ $e M_{N}(\operatorname{Diff}(\mathscr{V})) e$ is also closed under taking formal adjoints.

Similarly, we obtain the following easy consequence.

COROLlaRY 4.30. If $E_{0}, E_{1} \rightarrow M$ are Hermitian vector bundles, then the adjoint of an operator $P \in \operatorname{Diff}\left(\mathscr{V} ; E_{0}, E_{1}\right)$ is in $\operatorname{Diff}\left(\mathscr{V} ; E_{1}, E_{0}\right)$.

Proof. Write $E:=E_{0} \oplus E_{1}$ and use the resulting natural matrix notation for operators in $\operatorname{Diff}(\mathscr{V} ; E)$.

5. Geometric operators. In this section, we see that the Hodge Laplacian $\left(d+d^{*}\right)^{2}$ on forms and the classical Dirac operator on a Riemannian (spin) manifold $M_{0}$ with a Lie structure at infinity $(M, \mathscr{V})$ are differential operators generated by $\mathscr{V}=\Gamma(A)$. (See also [45] for some similar results.)

Both the classical Dirac operator and $d+d^{*}$ are generalized Dirac operators. We will show that any generalized Dirac operator is a differential operator generated by $\mathscr{V}$. Our approach follows closely that in [28].

5.1. Hodge Laplacians. Recall from Example 2.19 that the de Rham differential defines an element $d \in \operatorname{Diff}\left(\mathscr{V} ; \Lambda^{p} A^{*}, \Lambda^{p+1} A^{*}\right)$. 
Proposition 5.1. On a Riemannian manifold $M_{0}$ with a Lie structure at infinity $(M, \mathscr{V})$, the Hodge-Laplace operator

$$
\Delta_{p}=d^{*} d+d d^{*}=\left(d+d^{*}\right)^{2} \in \operatorname{Diff}\left(\mathscr{V} ; \Lambda^{p} A^{*}\right),
$$

that is, it is a differential operator generated by $\mathscr{V}$.

Proof. This follows directly from Corollary 4.30 and the construction in Example 2.19 .

5.2. Principal bundles and connection 1-forms. Let $E \rightarrow M$ be a vector bundle of rank $k$ carrying a metric and an orientation. In this subsection, we will show that giving a metric $A^{*}$-valued connection on $E$ is equivalent to giving an $A^{*}$-valued connection 1 -form on the frame bundle of $E$. Our approach generalizes the case of Riemannian manifolds (see, e.g., [46, Chapter II, Section 4]), hence we will omit some details.

For simplicity, we will assume from now on that the vector bundle $A \rightarrow M$ is orientable.

Let $P$ be a principal $G$-bundle. We denote the Lie algebra of $G$ by $\mathfrak{g}$. The most important example will be the bundle of oriented orthonormal frames of the bundle $E$, denoted by $\pi_{P}: P_{S O}(E) \rightarrow M$, which is a principal $\mathrm{SO}(k)$-bundle. Differentiating the action of $G$ gives rise to the canonical map

$$
\mathfrak{g} \longrightarrow \Gamma(T P), \quad V \longmapsto \tilde{V} .
$$

DEFINITION 5.2. An $A^{*}$-valued connection 1 -form $\omega$ is a $\mathfrak{g} \otimes A^{*}$-valued 1 -form on $P_{\mathrm{SO}}(A)$ satisfying the compatibility conditions

$$
\omega(\tilde{V})=V, \quad g^{*} \omega=\operatorname{Ad}_{g^{-1}} \omega \quad \forall V \in \operatorname{so}(\mathfrak{g}) .
$$

If $\mathfrak{g} \subset \operatorname{so}(k)$, we write $\omega=\left(\omega_{i j}\right)$ with respect to the standard basis of so $(k)$. In particular, the $\omega_{i j}$ are $A^{*}$-valued 1-forms on $P_{\mathrm{SO}}(A)$ satisfying $\omega_{i j}=-\omega_{j i}$.

Here " $A^{*}$-valued" is in the sense of Definition 4.4. Any $A^{*}$-valued connection 1-form on $P$ gives rise to a $G$-invariant Ehresmann connection on the bundle $P$ via $\tau=\{V \in$ $\left.\pi_{P}{ }^{\#} A \mid \omega(V)=0\right\}$. It is easy to check that this yields a one-to-one correspondence between $G$-invariant Ehresmann connections and connection 1-forms.

Proposition 5.3. Let $E \rightarrow M$ be a vector bundle. For any $A^{*}$-valued connection 1form on $P_{\mathrm{SO}}(E)$, there is a unique metric connection on $E$ satisfying the formula

$$
\nabla e_{i}=\sum_{j=1}^{n} \mathscr{E} * \omega_{j i} \otimes e_{j},
$$

where $\mathscr{E}=\left(e_{1}, \ldots, e_{n}\right)$ is a local section of $P_{\mathrm{SO}}(E)$. Conversely, any metric connection on E arises from such an $A^{*}$-valued connection 1-form.

Note that $\mathscr{E} * \omega_{i j}$ is a well-defined $A^{*}$-valued 1-form on $M$.

The proof is straightforward and runs completely analogous to [46, Chapter II, Proposition 4.4 ] with ordinary 1 -forms replaced by $A^{*}$-valued 1 -forms. As a result, we can 
conclude that the Levi-Civita connection on $A$ determines an $\mathrm{SO}(n)$-invariant Ehresmann connection and an $A^{*}$-valued connection 1-form on $P_{\mathrm{SO}}(A)$.

5.3. Spin structures and spinors. The results of the previous subsection now allow us to define the classical Dirac operator in a coordinate-free definition manner.

DEFINITION 5.4. A spin structure on $(M, \mathscr{V})$ is given by a $\operatorname{Spin}(n)$-principal bundle $P_{\text {Spin }}$ over $M$ together with bundle map $\theta: P_{\text {Spin }} \rightarrow P_{\text {SO }}(A)$, that is, $\operatorname{Spin}(n) \rightarrow \operatorname{SO}(n)$ equivariant.

The (thick) pullback of any $\mathrm{SO}(n)$-invariant Ehresmann connection on the principal $\mathrm{SO}(n)$-bundle $P_{\mathrm{SO}}(A) \rightarrow M$ with respect to $A$ defines a $\operatorname{Spin}(n)$-invariant Ehresmann connection on $P_{\text {Spin }} \rightarrow M$ with respect to $A$. Similarly, by using the standard identification of the Lie algebra of $\mathrm{SO}(n)$ with the Lie algebra of $\operatorname{Spin}(n)$, any $A^{*}$-valued connection 1 -form on $P_{\mathrm{SO}}(A)$ pulls back to an $A^{*}$-valued connection 1-form on $P_{\text {Spin }}$.

DeFINITION 5.5. Let $P$ be a principal bundle with respect to the Lie group $G$. Let $\mathfrak{g}$ and $\mathfrak{h}$ denote the Lie algebras of $G$ and $H$. Let $\varrho: G \rightarrow H$ be an inclusion of Lie groups. Then any $A^{*}$-valued connection 1 -form on $P$ defines an induced $A^{*}$-valued connection 1-form on $P \times{ }_{\varrho} H$ via the formula

$$
[X ; Y] \longmapsto \varrho_{*}(\omega(X))+Y \quad \forall X \in T P, Y \in T H
$$

This definition does not depend on the choice of representative $[X ; Y]$ as the map is invariant under the action of $G$ on $P \times H$.

Now, let $\sigma_{n}: \operatorname{Spin}(n) \rightarrow \operatorname{SU}\left(\Sigma_{n}\right)$ be the complex spinor representation; for example, the restriction of an odd irreducible complex representation of the Clifford algebra on $n$-dimensional space [46]. The complex dimension of $\Sigma_{n}$ is $d_{n}:=2^{[n / 2]}$.

DEFINITION 5.6. Let $M_{0}$ be an $n$-dimensional Riemannian manifold with a Lie structure at infinity $(M, \mathscr{V}, g)$ carrying a spin structure $P_{\text {Spin }}(A) \rightarrow P_{\mathrm{SO}}(A)$. The spinor bundle is the associated vector bundle $\Sigma M:=P_{\text {Spin }}(A) \times_{\sigma_{n}} \Sigma_{n}$ on $M$.

Any metric $A^{*}$-valued connection on $A$ gives rise to an $A^{*}$-valued connection on $\Sigma M$ as follows: Proposition 5.3 defines an $A^{*}$-valued connection 1-form on $P_{\mathrm{SO}}(A)$ which can be pulled back to $P_{\text {Spin }}(A)$. With Definition 5.5 applied to $\varrho=\sigma_{n}: \operatorname{Spin}(n) \rightarrow \operatorname{SU}\left(d_{n}\right) \subset$ $\mathrm{SO}\left(2 d_{n}\right)$, we obtain an $A^{*}$-valued connection 1 -form on $P_{\text {Spin }}(A) \times_{\sigma_{n}} \mathrm{SO}\left(2 d_{n}\right)$ compatible with complex multiplication. Another application of Proposition 5.3 yields a complex $A^{*}$-valued connection on $\Sigma M$.

In particular, the Levi-Civita connection on $A$ defines then a metric connection on $\Sigma M$, the so-called Levi-Civita connection.

Recall that the spinor representation $\Sigma_{n}$ admits a $\operatorname{Spin}(n)$-equivariant linear map

$$
\mathbb{R}^{n} \otimes \Sigma_{n} \longrightarrow \Sigma_{n}, \quad X \otimes \varphi \longmapsto X \cdot \varphi
$$

satisfying

$$
(X \cdot Y+Y \cdot X+2 g(X, Y)) \cdot \varphi=0
$$


for all $X, Y \in \mathbb{R}^{n}$ and all $\varphi \in \Sigma_{n}$, the so-called Clifford multiplication relations. By forming the associated bundles, this gives rise to a bundle map $A \otimes \Sigma M \rightarrow \Sigma M$, called Clifford multiplication. Equation (5.7) is satisfied for all $X, Y \in A, \varphi \in \Sigma M$ in the same basepoint.

5.4. Generalized Dirac operators. We now discuss Clifford modules in our setting.

Definition 5.7. A Clifford module over $M$ is a complex vector bundle $W \rightarrow M$ equipped with a positive definite product $\langle\cdot, \cdot\rangle$, antilinear in the second argument, an $A^{*}$-valued connection $\nabla^{W}$, and a linear bundle map $A \otimes W \rightarrow W, X \otimes \varphi \mapsto X \cdot \varphi$ called Clifford multiplication such that

(1) $(X \cdot Y+Y \cdot X+2 g(X, Y)) \cdot \varphi=0$,

(2) $\nabla^{W}$ is metric, that is,

$$
\partial_{X}\langle\psi, \varphi\rangle=\left\langle\nabla_{X}^{W} \psi, \varphi\right\rangle+\left\langle\psi, \nabla_{X}^{W} \varphi\right\rangle
$$

for $X \in \Gamma(A), \varphi, \psi \in \Gamma(W)$,

(3) Clifford multiplication with vectors is skew-symmetric, that is,

$$
\langle X \cdot \psi, \varphi\rangle=\langle\psi, X \cdot \varphi\rangle
$$

for $\varphi, \psi \in \Gamma(W), X \in \Gamma(A)$,

(4) Clifford multiplication is parallel, that is,

$$
\nabla_{X}^{W}(Y \cdot \varphi)=\left(\nabla_{X}^{W} Y\right) \cdot \varphi+Y \cdot\left(\nabla_{X}^{W} \varphi\right)
$$

for $X \in \Gamma(A), Y \in \Gamma(A)$, and $\varphi \in \Gamma(W)$.

The generalized Dirac operator associated to a Clifford module $W$ is the first-order operator $\not^{W}$ obtained by the following composition:

$$
\begin{gathered}
\Gamma(W) \stackrel{\nabla^{W}}{\longrightarrow} \Gamma\left(W \otimes A^{*}\right) \stackrel{\mathrm{id} \otimes \#}{\longrightarrow} \Gamma(W \otimes A) \stackrel{\rightarrow}{ } \Gamma(W), \\
\not^{W}:=\cdot \circ(\operatorname{id} \otimes \#) \circ \nabla^{W} .
\end{gathered}
$$

The last map is Clifford multiplication and $\#: A^{*} \rightarrow A$ is the isomorphism given by $g$.

The principal symbol of any generalized Dirac operator is elliptic, as for any nonzero vector $X$, Clifford multiplication by $X$ defines an invertible element of End $(\Sigma M)$.

EXAmPLE 5.8. For any $p \in M$, we define the Clifford algebra $\mathrm{Cl}\left(A_{p}\right)$ as the universal commutative algebra generated by $A_{p}$ subject to the relation

$$
X \cdot Y+Y \cdot X+2 g(X, Y) 1=0 .
$$

Let $\mathrm{Cl}(A)$ be the Clifford bundle of $(A, g)$, that is, the bundle whose fiber at the point $p \in M$ is the Clifford algebra $\mathrm{Cl}\left(A_{p}\right)$. The $A^{*}$-valued connection on $A$ extends to an $A^{*}$ valued connection on $\mathrm{Cl}(A)$. Let $W=\mathrm{Cl}(A)$, equipped with the module structure given by left multiplication. After identifying with the canonical isomorphism $\mathrm{Cl}(A) \cong \Lambda^{*}(A)$, $e_{i_{1}} \cdots e_{i_{k}} \mapsto e_{i_{1}}^{b} \wedge \cdots \wedge e_{i_{1}}^{b}$ for an orthonormal basis $\left(e_{i}\right)$ with dual $\left(e_{i}^{b}\right)$, the generalized Dirac operator on this bundle is the de Rham operator $d+d^{*}$. 
EXAMPLE 5.9. If $M$ is spin, then the spinor bundle from Definition 5.6 is also a Clifford module. The corresponding Dirac operator is called the (classical) Dirac operator.

EXAMPLE 5.10. If $M$ is even dimensional and if $A$ carries a Kähler structure, then the Dolbeault operator $\sqrt{2}\left(\bar{\partial}+\bar{\partial}^{*}\right)$ acting on $(0, *)$-forms is a generalized Dirac operator.

EXAMPLE 5.11. Let $W$ be any complex vector bundle with a positive definite scalar product and a Clifford multiplication such that (2.1) and (2.11) of Definition 5.7 are satisfied. Then there is always a connection $\nabla^{W}$ on $W$ satisfying the compatibility conditions (2.3) and (2.17). This can be seen as follows: if $M$ is spin, then we can write

$$
W=\Sigma M \otimes V,
$$

where $V$ is isomorphic to the homomorphisms from $\Sigma M$ to $W$ that are $\mathrm{Cl}(A)$-equivariant. The bundle $V$ carries a compatible metric. After choosing any metric $A^{*}$-valued connection $\nabla^{V}$ on $V$, the product connection on $W$ satisfies (2.3) and (2.17).

If $M$ is not spin, the connection can be constructed locally on an open covering in the same way, and the locally defined connections can then be glued together to a globally defined connection by using a partition of unity, hence we obtain the statement.

For any two sections $\sigma_{1}$ and $\sigma_{2}$ of $W$, we let

$$
\left(\sigma_{1}, \sigma_{2}\right):=\int_{M}\left\langle\sigma_{1}, \sigma_{2}\right\rangle
$$

This expression is not always defined. However, it is a well-defined scalar product on generalized $L^{2}$-spinor fields, that is, generalized spinor fields with $\int_{M}\left\langle\sigma_{i}, \sigma_{i}\right\rangle<\infty$. It is also well defined if one of the sections $s_{i}$ or $s_{j}$ has compact support and the other is locally $L^{2}$.

For the benefit of the reader, we recall the following basic result (see, e.g., [28]).

Proposition 5.12 [28]. Generalized Dirac operators ID on complete Riemannian manifolds are formally selfadjoint and essentially selfadjoint. More concretely, for smooth sections $\sigma_{i}$,

$$
\left(\not D \sigma_{1}, \sigma_{2}\right)=\left(\sigma_{1}, \not D \sigma_{2}\right)
$$

if at least one of the sections $\sigma_{1}$ or $\sigma_{2}$ has compact support. The maximal extension and the minimal extension of $D$ coincide. In particular, $D$ extends uniquely to a selfadjoint operator densely defined on the $L^{2}$-sections of $W$.

For any choice of a connection as in the above theorem, the resulting Dirac operator is generated by $\mathscr{V}$.

THEOREM 5.13. Let $W \rightarrow M$ be a Clifford module. Then the Dirac operator on $W$ is generated by $\mathscr{V}$ :

$$
\not D^{W} \in \operatorname{Diff}(\mathscr{V} ; W)
$$


Proof. The Dirac operator is the composition of Clifford multiplication and the $A^{*}$ valued connection $\nabla^{W}$ on $W$. Clifford multiplication is a zero-order differential operator generated by $\mathscr{V}$. The $A^{*}$-valued connection $\nabla^{W}$ on $W$ is a first-order differential operator generated by $\mathscr{V}$. Hence, the Dirac operator is also a first-order differential operator generated by $\mathscr{V}$.

ACKNOWLEDGments. We thank Sergiu Moroianu for several discussions on the subject. Nistor was partially supported by the National Science Foundation (NSF) Grants DMS-9971951 and DMS 02-00808, and "Collaborative Research" Grant. Ammann was partially supported by The European Contract Human Potential Programme, and Research Training Networks HPRN-CT-2000-00101 and HPRN-CT-1999-00118.

\section{REFERENCES}

[1] K. Akutagawa and B. Botvinnik, Yamabe metrics on cylindrical manifolds, Geom. Funct. Anal. 13 (2003), no. 2, 259-333.

[2] B. Ammann, R. Lauter, and V. Nistor, Pseudodifferential operators on manifolds with a Lie structure at infinity, preprint, 2003, http://arXiv.org/abs/math.AP/0304044.

[3] _ Algebras of pseudodifferential operators on complete manifolds, Electron. Res. Announc. Amer. Math. Soc. 9 (2003), 80-87.

[4] B. Ammann, R. Lauter, V. Nistor, and A. Vasy, Complex powers and non-compact manifolds, to appear in Comm. Partial Differential Equations, http://arXiv.org/abs/math.OA/ 0211305.

[5] M. T. Anderson, Einstein metrics with prescribed conformal infinity on 4-manifolds, to appear in Adv. Math.

[6] M. T. Anderson, P. T. Chruściel, and E. Delay, Non-trivial, static, geodesically complete, vacuum space-times with a negative cosmological constant, J. High Energy Phys. (2002), no. $10,1-27$.

[7] L. Andersson and M. Dahl, Scalar curvature rigidity for asymptotically locally hyperbolic manifolds, Ann. Global Anal. Geom. 16 (1998), no. 1, 1-27.

[8] R. Bartnik, The mass of an asymptotically flat manifold, Comm. Pure Appl. Math. 39 (1986), no. 5, 661-693.

[9] N. Berline, E. Getzler, and M. Vergne, Heat Kernels and Dirac Operators, Grundlehren der Mathematischen Wissenschaften, vol. 298, Springer-Verlag, Berlin, 1992.

[10] H. Boualem and M. Herzlich, Rigidity at infinity for even-dimensional asymptotically complex hyperbolic spaces, Ann. Sc. Norm. Super. Pisa Cl. Sci. V 1 (2002), no. 2, 461-469.

[11] P. T. Chruściel and M. Herzlich, The mass of asymptotically hyperbolic Riemannian manifolds, Tech. Report 13, Département de Math., Univ. Tours, 2001.

[12] A. Connes, Sur la théorie non commutative de l'intégration, Algèbres d'opérateurs (Sém., Les Plans-sur-Bex, 1978), Lecture Notes in Math., vol. 725, Springer-Verlag, Berlin, 1979, pp. 19-143 (French).

[13] H. O. Cordes, Spectral Theory of Linear Differential Operators and Comparison Algebras, London Mathematical Society Lecture Note Series, vol. 76, Cambridge University Press, Cambridge, 1987.

[14] _ The Technique of Pseudodifferential Operators, London Mathematical Society Lecture Note Series, vol. 202, Cambridge University Press, Cambridge, 1995.

[15] H. O. Cordes and S. H. Doong, The Laplace comparison algebra of spaces with conical and cylindrical ends, Pseudodifferential Operators (Oberwolfach, 1986), Lecture Notes in Math., vol. 1256, Springer-Verlag, Berlin, 1987, pp. 55-90.

[16] H. O. Cordes and R. C. McOwen, The $C^{*}$-algebra of a singular elliptic problem on a noncompact Riemannian manifold, Math. Z. 153 (1977), no. 2, 101-116. 
[17]

Math. 70 (1977), no. 1, 133-141.

[18] M. Crainic and R. L. Fernandes, Integrability of Lie brackets, Ann. of Math. (2) 157 (2003), no. 2, 575-620.

[19] C. Ehresmann, Les connexions infinitésimales dans un espace fibré différentiable, Colloque de Topologie (Espaces Fibrés), Bruxelles, 1950, Georges Thone, Liège, 1951, pp. 2955 (French).

[20] J. Eichhorn, Riemannsche Mannigfaltigkeiten mit einer zylinderähnlichen Endenmetrik [Riemannian manifolds with cylinder-like end metrics], Math. Nachr. 114 (1983), 23-51 (German).

[21] , Elliptic differential operators on noncompact manifolds, Seminar Analysis of the Karl-Weierstrass-Institute of Mathematics (Berlin, 1986/1987), Teubner-Texte Math., vol. 106, Teubner, Leipzig, 1988, pp. 4-169.

, The boundedness of connection coefficients and their derivatives, Math. Nachr. 152 (1991), 145-158.

J. Eichhorn, J. Fricke, and A. Lang, Metrics conformally equivalent to bounded geometry, Rend. Circ. Mat. Palermo (2) Suppl. (1996), no. 43, 103-110.

C. L. Epstein, R. B. Melrose, and G. A. Mendoza, Resolvent of the Laplacian on strictly pseudoconvex domains, Acta Math. 167 (1991), no. 1-2, 1-106.

S. Evens, J.-H. Lu, and A. Weinstein, Transverse measures, the modular class and a cohomology pairing for Lie algebroids, Quart. J. Math. Oxford Ser. (2) 50 (1999), no. 200, 417-436.

M. P. Gaffney, The harmonic operator for exterior differential forms, Proc. Natl. Acad. Sci. USA 37 (1951), 48-50.

S. Gallot, D. Hulin, and J. Lafontaine, Riemannian Geometry, 2nd ed., Universitext, SpringerVerlag, Berlin, 1990.

M. Gromov and H. B. Lawson Jr., Positive scalar curvature and the Dirac operator on complete Riemannian manifolds, Inst. Hautes Études Sci. Publ. Math. (1983), no. 58, 83196.

E. Hebey, Sobolev Spaces on Riemannian Manifolds, Lecture Notes in Mathematics, vol. 1635, Springer-Verlag, Berlin, 1996.

M. Herzlich, Scalar curvature and rigidity of odd-dimensional complex hyperbolic spaces, Math. Ann. 312 (1998), no. 4, 641-657.

P. J. Higgins and K. C. H. Mackenzie, Fibrations and quotients of differentiable groupoids, J. London Math. Soc. (2) 42 (1990), no. 1, 101-110.

L. Hörmander, The Analysis of Linear Partial Differential Operators. I, Grundlehren der Mathematischen Wissenschaften, vol. 256, Springer-Verlag, Berlin, 1983. , The Analysis of Linear Partial Differential Operators. III, Grundlehren der Mathematischen Wissenschaften, vol. 274, Springer-Verlag, Berlin, 1985.

D. D. Joyce, Compact Manifolds with Special Holonomy, Oxford Mathematical Monographs, Oxford University Press, Oxford, 2000. , Asymptotically locally Euclidean metrics with holonomy SU(m), Ann. Global Anal. Geom. 19 (2001), no. 1, 55-73. , Quasi-ALE metrics with holonomy $\mathrm{SU}(m)$ and $\mathrm{Sp}(m)$, Ann. Global Anal. Geom. 19 (2001), no. 2, 103-132.

M. Karoubi, K-Theory. An Introduction, Grundlehren der Mathematischen Wissenschaften, vol. 226, Springer-Verlag, Berlin, 1978.

V. A. Kondratiev, Boundary value problems for elliptic equations in domains with conical or angular points, Trans. Moscow Math. Soc. 16 (1967), 227-313.

Yu. A. Kordyukov, $L^{p}$-theory of elliptic differential operators on manifolds of bounded geometry, Acta Appl. Math. 23 (1991), no. 3, 223-260. 
[40] V. A. Kozlov, V. G. Mazỳa, and J. Rossmann, Spectral Problems Associated with Corner Singularities of Solutions to Elliptic Equations, Mathematical Surveys and Monographs, vol. 85, American Mathematical Society, Rhode Island, 2001.

[41] R. Lauter, Pseudodifferential analysis on conformally compact spaces, Mem. Amer. Math. Soc. 163 (2003), no. 777, 1-92.

[42] R. Lauter, B. Monthubert, and V. Nistor, Pseudodifferential analysis on continuous family groupoids, Doc. Math. 5 (2000), 625-655.

[43] R. Lauter and S. Moroianu, Fredholm theory for degenerate pseudodifferential operators on manifolds with fibered boundaries, Comm. Partial Differential Equations 26 (2001), no. 1-2, 233-283.

[44] R. Lauter and V. Nistor, Pseudodifferential analysis on groupoids and singular spaces, Mainz, preprint, 1999.

[45] _ Analysis of geometric operators on open manifolds: a groupoid approach, Quantization of Singular Symplectic Quotients (N. P. Landsman, M. Pflaum, and M. Schlichenmaier, eds.), Progr. Math., vol. 198, Birkhäuser, Basel, 2001, pp. 181-229.

[46] H. B. Lawson Jr. and M.-L. Michelsohn, Spin Geometry, Princeton Mathematical Series, vol. 38, Princeton University Press, New Jersey, 1989.

[47] J. Lee, Fredholm operators and Einstein metrics on conformally compact manifolds, preprint, 2001, http://arXiv.org/abs/math.DG/0105046.

[48] M. Lesch, Operators of Fuchs Type, Conical Singularities, and Asymptotic Methods, TeubnerTexte zur Mathematik, vol. 136, B. G. Teubner Verlagsgesellschaft mbH, Stuttgart, 1997.

[49] K. Mackenzie, Lie Groupoids and Lie Algebroids in Differential Geometry, London Mathematical Society Lecture Note Series, vol. 124, Cambridge University Press, Cambridge, 1987.

[50] R. R. Mazzeo, Elliptic theory of differential edge operators. I, Comm. Partial Differential Equations 16 (1991), no. 10, 1615-1664.

[51] R. R. Mazzeo and R. B. Melrose, Meromorphic extension of the resolvent on complete spaces with asymptotically constant negative curvature, J. Funct. Anal. 75 (1987), no. 2, 260-310.

[52] R. R. Mazzeo and F. Packard, Poincaré-Einstein metrics and the Schouten tensor, preprint, 2001, http://arXiv.org/abs/math.DG/0105171.

[53] R. Melrose and V. Nistor, $K$-theory of $C^{*}$-algebras of b-pseudodifferential operators, Geom. Funct. Anal. 8 (1998), no. 1, 88-122.

[54] R. B. Melrose, Transformation of boundary problems, Acta Math. 147 (1981), no. 3-4, 149236.

[55] _ Pseudodifferential operators, corners and singular limits, Proceedings of the International Congress of Mathematicians, Vol. I, II (Kyoto, 1990), Mathematical Society of Japan, Tokyo, 1991, pp. 217-234.

[56] _ The Atiyah-Patodi-Singer Index Theorem, Research Notes in Mathematics, vol. 4, A K Peters, Massachusetts, 1993.

[57] _ Geometric Scattering Theory, Stanford Lectures, Cambridge University Press, Cambridge, 1995.

[58] M. Mitrea and V. Nistor, Boundary value problems and layer potentials on manifolds with cylindrical ends, preprint, 2002.

[59] B. Monthubert, Pseudodifferential calculus on manifolds with corners and groupoids, Proc. Amer. Math. Soc. 127 (1999), no. 10, 2871-2881.

[60] S. Moroianu, Adiabatic limits of eta and zeta functions of elliptic operators, preprint, 2002.

[61] _ Higher residues on the algebra of adiabatic pseudodifferential operators, Ph.D. thesis, Massachusetts Institute of Technology, Massachusetts, 1999.

[62] W. Müller, On the $L^{2}$-index of Dirac operators on manifolds with corners of codimension two. I, J. Differential Geom. 44 (1996), no. 1, 97-177. 
[63] V. Nistor, A. Weinstein, and P. Xu, Pseudodifferential operators on differential groupoids, Pacific J. Math. 189 (1999), no. 1, 117-152.

[64] C. Parenti, Operatori pseudo-differenziali in $R^{n}$ e applicazioni, Ann. Mat. Pura Appl. (4) 93 (1972), 359-389 (Italian).

[65] T. H. Parker and C. H. Taubes, On Witten's proof of the positive energy theorem, Comm. Math. Phys. 84 (1982), no. 2, 223-238.

[66] J. Råde, Elliptic uniformly degenerate operators, preprint, 1999.

[67] J. Roe, An index theorem on open manifolds. I, J. Differential Geom. 27 (1988), no. 1, 87113.

[68] W. Roelcke, Über den Laplace-Operator auf Riemannschen Mannigfaltigkeiten mit diskontinuierlichen Gruppen, Math. Nachr. 21 (1960), 131-149 (German).

[69] R. Schoen, Conformal deformation of a Riemannian metric to constant scalar curvature, J. Differential Geom. 20 (1984), no. 2, 479-495.

[70] E. Schrohe, Spectral invariance, ellipticity, and the Fredholm property for pseudodifferential operators on weighted Sobolev spaces, Ann. Global Anal. Geom. 10 (1992), no. 3, 237254.

[71] E. Schrohe and B.-W. Schulze, Boundary value problems in Boutet de Monvel's algebra for manifolds with conical singularities. I, Pseudo-Differential Calculus and Mathematical Physics, Math. Top., vol. 5, Akademie Verlag, Berlin, 1994, pp. 97-209.

[72] _ Boundary value problems in Boutet de Monvel's algebra for manifolds with conical singularities. II, Boundary Value Problems, Schrödinger Operators, Deformation Quantization, Math. Top., vol. 8, Akademie Verlag, Berlin, 1995, pp. 70-205.

[73] B.-W. Schulze, Boundary Value Problems and Singular Pseudo-Differential Operators, Pure and Applied Mathematics, John Wiley \& Sons, Chichester, 1998.

[74] _ Operator algebras with symbol hierarchies on manifolds with singularities, Approaches to Singular Analysis (Berlin, 1999), Oper. Theory Adv. Appl., vol. 125, Birkhäuser, Basel, 2001, pp. 167-207.

[75] M. A. Shubin, Spectral theory of elliptic operators on noncompact manifolds, Astérisque (1992), no. 207, 35-108.

[76] M. E. Taylor, Gelfand theory of pseudo differential operators and hypoelliptic operators, Trans. Amer. Math. Soc. 153 (1971), 495-510.

[77] A. Vasy, Propagation of singularities in many-body scattering, Ann. Sci. École Norm. Sup. (4) 34 (2001), no. 3, 313-402.

[78] A. Weinstein, Groupoids: unifying internal and external symmetry. A tour through some examples, Notices Amer. Math. Soc. 43 (1996), no. 7, 744-752.

Bernd Ammann: Fachbereich Mathematik, Universität Hamburg, Bundesstraße 55, D-20146 Hamburg, Germany

E-mail address: ammann@math . uni-hamburg.de

Robert Lauter: Fachbereich Mathematik, Universität Mainz, D-55099 Mainz, Germany

E-mail address: 1auter@mathematik.uni-mainz.de

Victor Nistor: Department of Mathematics, Pennsylvania State University, University Park, PA 16802, USA

E-mail address: nistor@math.psu.edu 


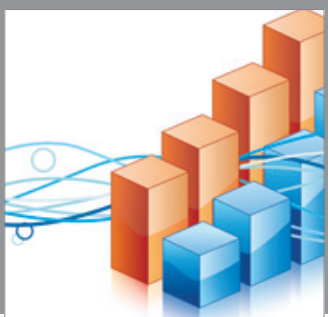

Advances in

Operations Research

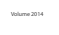

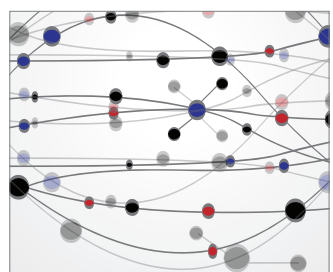

\section{The Scientific} World Journal
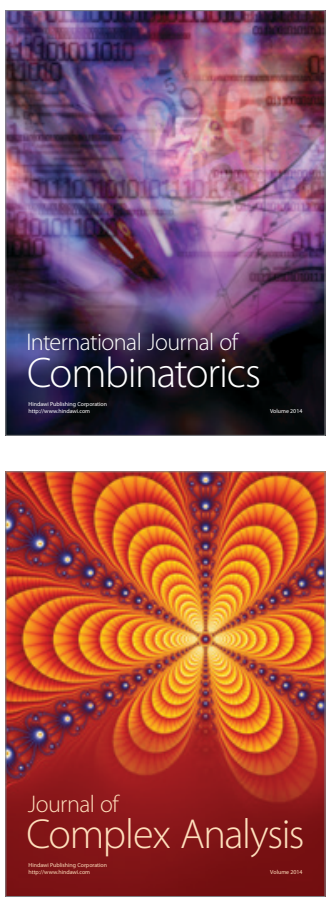

International Journal of

Mathematics and

Mathematical

Sciences
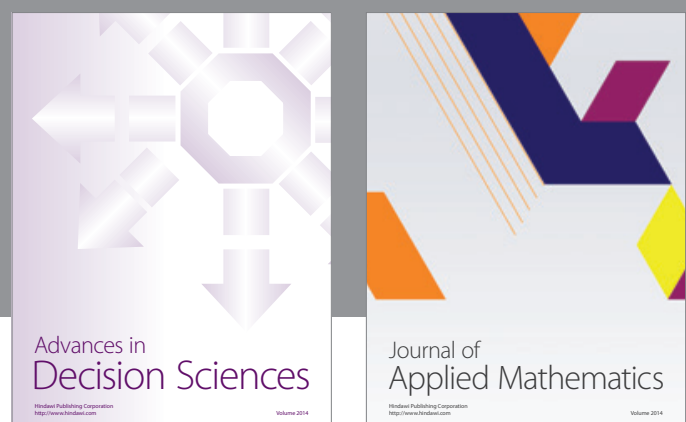

Journal of

Applied Mathematics
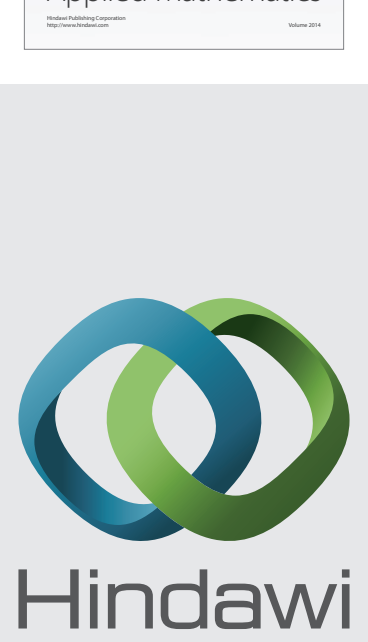

Submit your manuscripts at http://www.hindawi.com
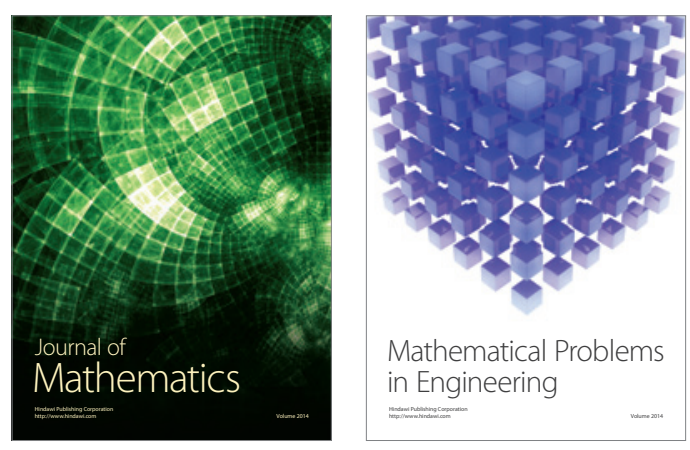

Mathematical Problems in Engineering
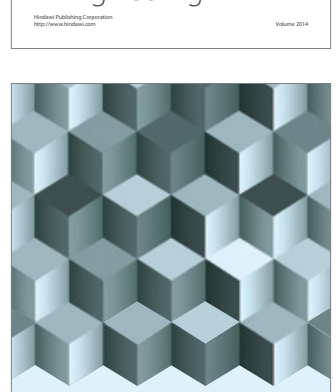

Journal of

Function Spaces
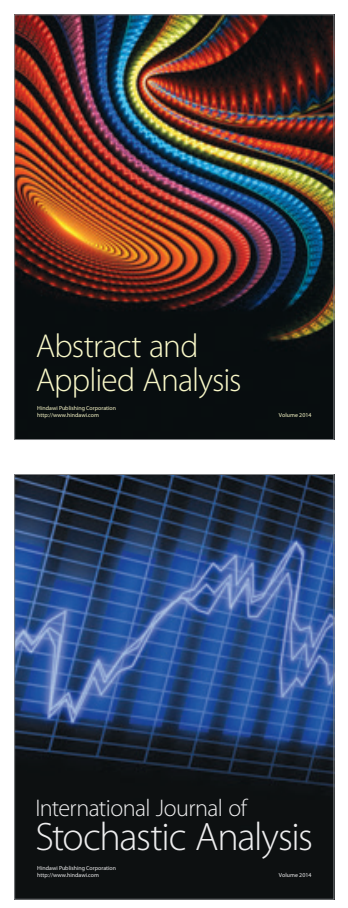

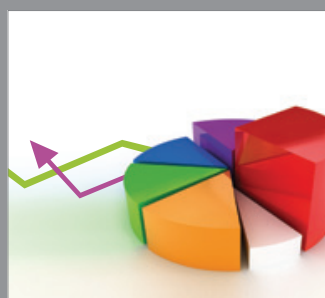

ournal of

Probability and Statistics

Promensencen
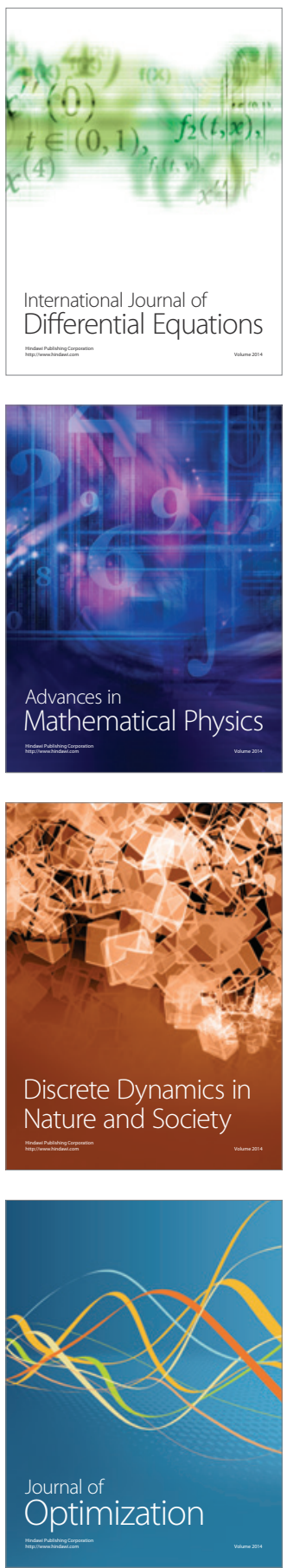